\title{
WHY DO WE NEED 6G?
}

\author{
Riccardo Bassoli ${ }^{1}$, Frank H.P. Fitzek ${ }^{1,2}$, Emilio Calvanese Strinati ${ }^{3}$ \\ ${ }^{1}$ Deutsche Telekom Chair of Communication Networks, Institute of Communication Technology, Faculty of Electrical and \\ Computer Engineering, Technische Universität Dresden, Dresden, Germany, ${ }^{2}$ Centre for Tactile Internet with \\ Human-in-the-Loop (CeTI), Cluster of Excellence, Dresden, Germany, ${ }^{3}$ CEA Leti, 17 rue des Martyres, 38000 Grenoble, \\ France,
}

NOTE: Corresponding author: Riccardo Bassoli, riccardo.bassoli@tu-dresden.de

\begin{abstract}
The study and design of 5G seems to have reached its end and 5 G communication systems are currently under deployment. In parallel, $5 \mathrm{G}$ standardization is as Release 16, which is going to complete the definition and the design guidelines of the $5 G$ radio access network. Because of that, the interest of the scientific and industrial communities has already started focusing on the future $6 G$ communication networks. The preliminary definition of future technology trends towards 2030, given by major standardization bodies, and the flagship $6 G$ projects worldwide have started proposing various visions about what $6 G$ will be. Side by side, various scientific articles, addressing the initial characterisation of $6 G$, have also been published. However, considering the promises of $5 G$, can $6 G$ represent a significant technological advancement to justify a so-called new generation? In fact, now, $5 G$ softwarized networks may just imply continuous network software upgrades (as it happens for the Internet) instead of new generations every ten years. This article starts describing the main characteristics that made $5 G$ a breakthrough in telecommunications, also briefly introducing the network virtualisation and computing paradigms that have reformed telecommunications. Next, by providing rigorous definition of the terminology and a survey of the principal $6 G$ visions proposed, the paper tries to establish important motivations and characteristics that can really justify the need for and the novelty of future $6 G$ communication networks.
\end{abstract}

Keywords - 3D networking, 6G, beyond 5G, beyond Shannon, digital twin networking, goal oriented communications, key performance indicators, native artificial intelligence, semantic communications

\section{INTRODUCTION}

Wireless cellular communication networks have seen the rise of a so-called new generation approximately every ten years. Each generation has always provided a disruptive technological advancement and societal change. The first generation (1G) first introduced analogue wireless cellular technologies. On the other hand, the second generation (2G) was the first wireless cellular network based on digital technologies. Next, the third generation (3G) not only provided the support for wireless voice telephony but also for Internet access, video calls, and mobile television. Finally, the fourth generation (4G) was the first mobile cellular network with full support for Internet Protocol (IP) and access to Internet services. With the fifth generation (5G), the gateway to digitisation in industry has been pushed wide open. $5 \mathrm{G}$ has been addressing the support to controlling machines, and to give connectivity to several applications besides industry, such as agriculture, the construction sector, the energy sector with so-called smart grids, and the Internet of Things (IoT) also possible in real time.

Before 5G, communication networks mainly focused on providing continuously higher data rates to offer highresolution streaming services. On the other hand, $5 \mathrm{G}$ has been targeting the support for millions of devices at very low latency. Low latency combined with resilience are the key to realise the remote control of machines, and it also allows people and machines to co-work in the same realvirtual environment. That is why the setup of $5 \mathrm{G}$ mobile systems is so important, even if low latency will only be realised with the next $5 \mathrm{G}$ releases. To this end, the leader of 5G standardization body, the 3rd Generation Partnership Project (3GPP), has already prepared a clear road map with the next steps together with the International Telecommunication Union (ITU). So it is not the case that the development of $5 \mathrm{G}$ is complete. The $5 \mathrm{G}$ network is continuously evolving and will be adapted and optimised to the tasks ahead.

However, 5G is not just an agnostic bit pipe, describing the simple passing of bits, as in previous generations. The new approach is achieved through the concept of softwarization. While its predecessors came in the form of dedicated hardware, 5G is mainly based on tailored software solutions running on general-purpose hardware, referring to low-cost, interchangeable and readily available products. Various softwarization concepts such as Software-Defined Radio (SDR) or Software-Defined Networking (SDN) are going to enable network operators to keep both Operational Expenditure (OPEX) and Capital Expenditure (CAPEX) low [1].

Recently, the attention and the effort towards the next generation $(6 \mathrm{G})$ has started both in the scientific, public, 
and private communities. Some consortia have already started working on the definition and characterisation of future $6 \mathrm{G}$ communication networks, preparing the ground for its standardization by 2030. In Europe, some of the major projects are the EU Flagship Hexa$X$ [2], the 5GPPP CORDIS RISE-6G [3], and the 5GPPP RIA DEDICAT 6G [4]. In parallel, US has also started the Next G Alliance [5]. All those results are expected to be contributed towards the ITU Radiocommunication Sector (ITU-R), which has initiated the work on "systems beyond IMT-2020" (see Section 4).

However, various promises made by $5 \mathrm{G}$ are still not satisfied and there is the feeling in part of the community that $6 \mathrm{G}$ could just be a $5 \mathrm{G}+$. Moreover, network softwarization brought $5 \mathrm{G}$ closer to the Internet community, which does not use any 'generation' terminology, but it provides continuous network upgrades and software updates. Next, $5 \mathrm{G}$ has already promised support to many verticals, which are still waiting to receive the promised low-latency reliable connectivity and almost continuous service availability. In such conceptual/technical still 'liquid' and changing situation, this article tries to state some important conceptual, terminological, and technical characteristics and guidelines that will make 6G. In this sense, this paper will try to give a solid answer to questions such as What can $6 G$ be? Do we really need 6G? Because of that, the following provides a significant analysis of the literature and of the evolution of the characteristics of $5 \mathrm{G}$ and $6 \mathrm{G}$, in order to propose some stable reasons for justifying the current standardization and research effort towards 6G.

First, Section 2 briefly introduces the path towards $4 \mathrm{G}$, and origins of the concepts of virtualisation and cloud computing. Next, Section 3 describes the advent of 5G, its characteristics, requirements, and the kinds of services it targets. With Section 4, the objective is to survey a significant part of the literature in order to give a review of the current proposed metrics, characteristics, and verticals proposed within 6G. This section is important to state the conceptual basics, which are critically discussed in Section 5 in order to highlight the main fundamental aspects of $6 \mathrm{G}$ together with the issues and the trade offs that will require significant investigation withing the scientific community and the industry.

\section{THE COMMUNICATION PANORAMA BEFORE 5G}

\subsection{The standardization and architecture of wireless cellular networks}

In September 2014, Release 8 of the standard 3GPP System Architecture Evolution Specification (SAES) was published. This is the first document introducing the concept of Evolved Packet Core (EPC) [6] - more generally labelled Evolved Packet System (EPS). In fact, the term EPS represents the end-to-end system, which mainly consists of User Equipment (UE), Evolved Universal Terrestrial Radio
Access Network (E-UTRAN), and the core network. The term E-UTRAN means the Radio Access Network (RAN) of Long Term Evolution (LTE) together with UMTS Terrestrial Radio Access Network (UTRAN) and GSM RAN (GERAN), interconnected via the EPC.

The Global System for Mobile Communications (GSM) and the Universal Mobile Telecommunications System (UMTS) represents the 2nd and 3rd Generation of wireless cellular networks, standardization efforts of European Telecommunications Standards Institute (ETSI) and 3GPP respectively. As it is possible to notice in the context of wireless cellular networks, the term 'generation' (identified by '. G') has been representing a significant change in the characteristics and performances of the RAN - 1G and $2 \mathrm{G}$, or in how different RANs are interconnected, $3 \mathrm{G}$ and $4 \mathrm{G}$. The switch of generations has maintained a periodicity of about ten years.

At the end of the 1990s, the deployment of 3G started, under the specifications of ITU International Mobile Telecommunications-2000 (IMT-2000). The scope of 3G was to support a variety of mobile broadband services not only wireless voice telephony but also mobile Internet access, video telephony, and mobile television. With this generation, data started becoming the core aspect of wireless cellular communications. Next, this standard was also guaranteeing global mobility via a harmonisation of the frequency bands, which set some common bands worldwide.

Finally, the deployment of the 4th Generation-LTE started around 2009. The objective of $4 \mathrm{G}$ was to improve the performance at the RAN also extending the initial focus on data started with $3 \mathrm{G}$, providing a wireless cellular network architecture with full access to the Internet. The definition of the EPC provided a core network, which unified the different radio access technologies under a single infrastructure. Additionally, LTE also started the integration with non-3GPP radio access technologies. This is clearly visible in the part of the standard focused on interworking between LTE and Wireless Local Area Network (WLAN) - embodied by the Institute of Electrical and Electronics Engineers (IEEE) 802.11 standard [6],[7].

Fig. 1 depicts the structure of the $4 \mathrm{G}$ RAN and its EPC. There are various logical blocks in the EPS, each one grouping logical nodes that interwork to provide a specific set of functions in the network. From the RAN perspective, all the 2G-4G RANs coexist and connect to the EPC. There is also the domain called 'non-3GPP access networks', which denotes any packet data access network that is not defined by 3GPP standards such as IEEE 802.11 WLAN and WiMAX. Side by side, the core network also consists of multiple domains such as circuit core, packet core and IP-Multimedia Subsystem (IMS). The user management domain provides coordinated subscriber information and supports roaming and mobility between and 


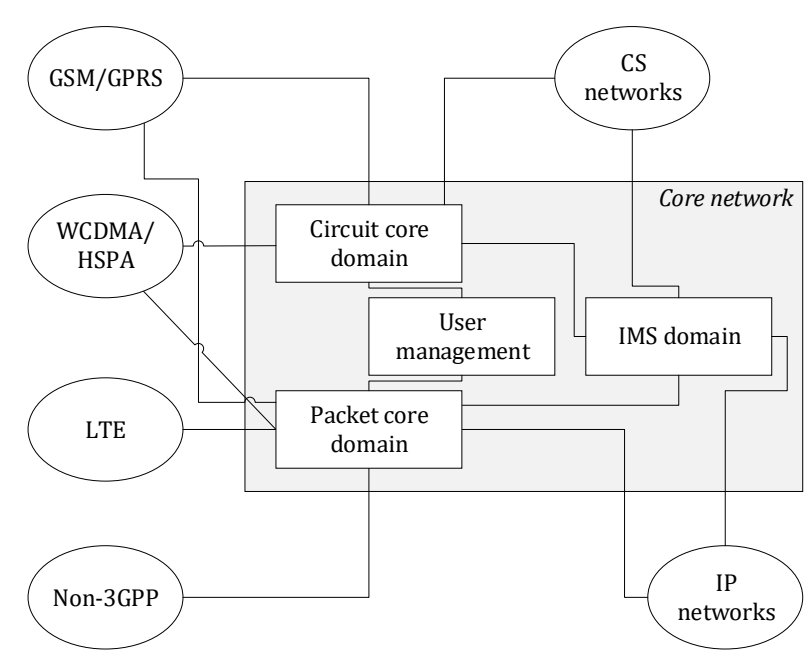

Fig. 1 - Domains in the 3GPP architecture of the evolved packet system [6].

within the different domains. The circuit core domain consists of nodes and functions that provide support for circuit-switched services over GSM and Wideband CodeDivision Multiple Access (WCDMA).

Next, the packet core domain consists of nodes and functions that provide support for packet-switched services. The packet core domain also provides functions for management of the Quality-of-Service (QoS). The IMS includes equipment and functions that provide support for mul-timedia sessions. In the LTE RAN there are base sta-tions called eNodeB. All eNodeBs are connected to at least one Mobility Management Entity (MME), which han-dles all control plane signalling. The IP packets flowing to and from mobile devices are handled by two logical nodes called the Serving Gateway (S-GW) and the Packet Data Network Gateway (PDN-GW). The S-GW is the an-chor point for intraLTE mobility, as well as for mobil-ity between GSM/ General Packet Radio Service (GPRS), WCDMA/High Speed Packet Access (HSPA) and LTE. The S-GW also buffers downlink IP packets destined for ter-minals that happen to be in idle mode, as well as supports transport level QoS through marking IP packets. On the other hand, the PDN-GW is the point of interconnection to external IP networks. The PDN-GW includes functionality for IP address allocation, charging, packet filtering and policy-based control of user-specific IP flows. The PDN$\mathrm{GW}$ also has a key role in supporting QoS for IP services.

The latest releases of LTE also included new connection paradigms that were becoming quite popular in the technical and scientific community such as relaying and Device-to-Device (D2D). LTE also opened the way to cellular networks with base stations of different coverage size. This meant the coexistence of large and small cells with the deployment of the Relay Node (RN), a lower power 3GPP base station enhancing the coverage at the cell's edge. The RN is wirelessly connected to a Donor eNodeB (DeNB), so that the donor cell provides shared radio resources between the UEs (not served by the DeNB) and the DeNB. In parallel, the D2D paradigm [8], [9] was mentioned in LTE Advanced in order to enable direct communications between UEs, without going through the eNodeB and the core network. However, the implementation and diffusion of D2D communications has remained in a very preliminary phase. With the advent of LTE, there was also a significant improvement for public safety networks [10]. The original limitations to voice services began to expand to more general broadband communications such as multimedia sharing, video calls, and livevideo streaming. Finally, even if some preliminary proposals were provided for deploying LTE in aerial networks [11], the LTE has remained a fully terrestrial wireless cellular network.

\subsection{The dawn of cloud computing and the standardization of softwarized networks}

The vision of $5 \mathrm{G}$ was not an agnostic and independent design project and standardization effort. For the first time in the panorama of telecommunications, a wireless cellular network absorbed the very novel paradigms in computing and networking in order to move towards a real breakthrough with the previous evolution. Concepts like cloud computing, and SDN and Network Function Virtualisation (NFV), radically shaped the idea of a communication network, as previously meant. This revolution in communications also inherently changed the procedure of standardization of wireless cellular networks. From $5 \mathrm{G}$ onwards, the standardization of wireless networks has become a global and collective effort of several standardization bodies, collaboratively and complementary working towards the same target. Fig. 2 depicts the standardization effort of 5G, led by $3 \mathrm{GPP}$ and ITU, and cooperatively supported by the major standardization bodies worldwide.

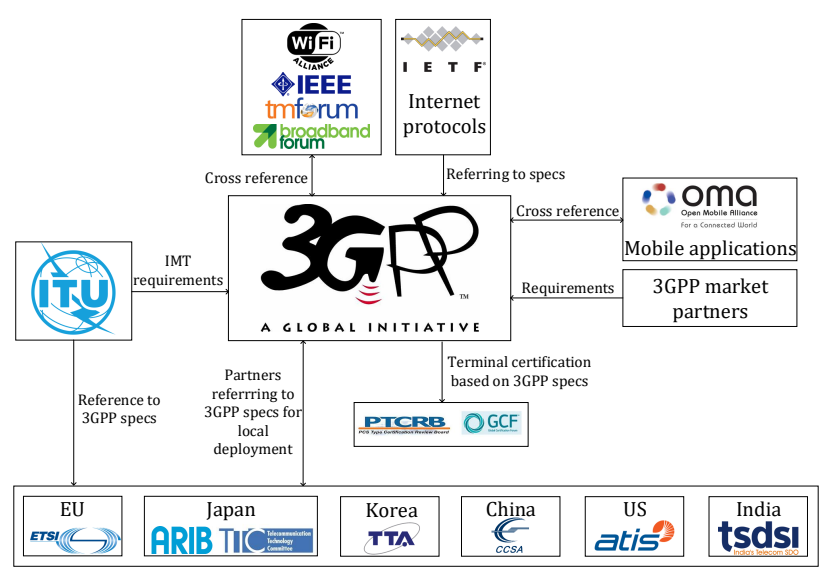

Fig. 2 - Structure of the standardization effort of 5G [1]. 
First, the vision of outsourcing and centralising computing has been the main driver for a paradigm-shift of innetwork computing. The so-called cloud computing originally started and evolved during the second half of the 21st century, when the computing hardware and the Internet grew. However, around 2008, the term cloud computing became popular, due to the maturity of the technologies to provide remote access to computing equipment in data centres, together with the growth of private entities such as Salesforce.com, Google, and Amazon Web Services. Cloud computing means a "[...] method of running application software and storing related data in central computer systems and providing customers or other users access to them through the Internet. [...]" [12]. As it is possible to guess, this technology created the bridge between big data centres and computing, and wireless cellular networks. Within cloud computing, it is possible to identify a taxonomy of services, which can be grouped into three main paradigms, according to what is made available: Infrastructure-as-a-Service (IaaS) for remote hardware access, Platform-as-a-Service (PaaS) for software platform access, and Software-as-a-Service (SaaS) for remote software access.

Next, the idea of network virtualisation (and more precisely softwarization) was changing the way networks had been intended by then, by transposing any network function, protocol, operation, etc. from dedicated hardware to software, running on general-purpose hardware. If we generally consider a network, its functions can logically be grouped into two planes, the so-called data (or user) and control planes. Around 2010, the original visions of programmable networks and decoupling data and control planes became reality via the first developments of SDN, led by the nonprofit consortium Open Network Foundation (ONF).

In order to achieve this decoupling, SDN requires three main entities [1]:

- a centralised SDN controller, which changes the entries in the flow tables of SDN switches according to either static or dynamic algorithms, in order to manage the paths of packets among end users (computers);

- SDN switches, that contains the flow tables to manage the routing of messages from source(s) to destination(s);

- a control protocol (e.g. Openflow), which enables the communication between controller and switches, while also analysing and modifying the flow tables within the SDN switches.

Currently, an Open Network Operating System (ONOS) is leading the design and implementation of an opensource SDN controller. This controller can also be softwarized and outsourced to the cloud, in a centralised or distributed manner to improve for example resilience and scalability. In 2020, ONOS started the $\mu$ ONOS project aimed at designing and realising a new generation of open-source SDN controllers. While original SDN controllers (either centralised or distributed) have been 'monolithic' entities, the $\mu$ ONOS controller will be based on micro-services. This important change will permit the split of the controller into various subfunctions. Each subfunction will be responsible for a certain control operation and/or configuration and/or management function of the SDN network. In $\mu$ ONOS, the so-called service orchestrator (i.e. Kubernetes) manages each micro-service, running in a software container.

In 2012, NFV was proposed by the industrial community (i.e. AT\&T, BT, CenturyLink, China Mobile, Deutsche Telekom, KDDI, NTT, Orange, Telecom Italia, Telefonica, Telstra and Verizon) with the publication of the white paper [13]. Next, these operators identified ETSI as the standardization body to undertake the standardization effort of this paradigm. In NFV, a network element/function/entity/etc. is no longer hardwaredependent but it is a complete software element, running on any general-purpose hardware within the network. The NFV original architecture consists of three main layers [1]:

- Physical resources, which are "A physical asset for computation, storage and transport (e.g. switch, router, antenna, etc.)" [14];

- Virtual resources, that are "An abstraction of physical or logical resource, which may have different characteristics from the physical or logical resource and whose capability may be not bound to the capability of the physical or logical resource" [14];

- Services, which are the virtual network functions, running in software environments such as virtual machines, containers, etc. [15]

Next, there is a transverse management layer called Management and Orchestration (MANO), which handles the provisioning of virtual network functions, their configuration, placement, orchestration, and management of physical and virtual resources. This layer contains the socalled orchestrator.

After the previous design and standardization efforts, ETSI started focusing on the unification of SDN and NFV in a unique architecture. The outcome was the ETSI MANO SDN-NFV architecture, which is depicted in Fig. 3. The whole architecture first consists of four main blocks such as:

- the Network Management System (NMS), responsible for management of the virtual network;

- the Network Function Virtualization Infrastructure (NFVI), the set of resources (physical or virtualized) that are used to run and to connect virtual network functions; 


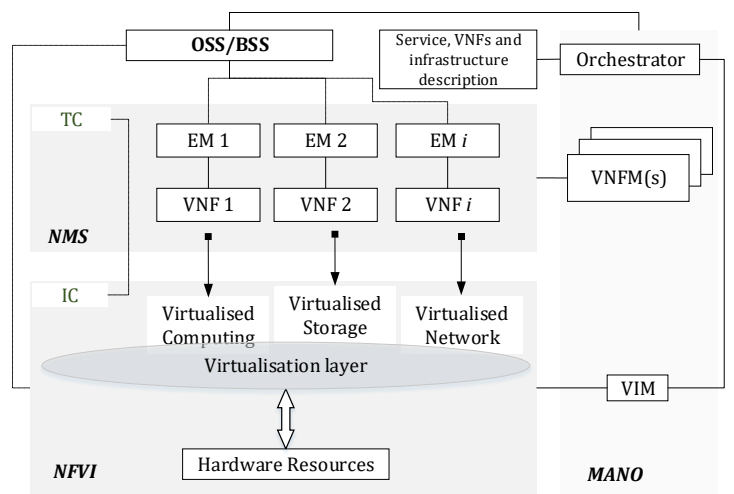

Fig. 3 - ETSI MANO SDN-NFV architecture.

- the MANO;

- the Operation/Business Support Scheme (OSS/BSS), the set of applications (e.g., sysytem level and management) that are used by service providers to provide network services.

First, the NMS includes the Tenant SDN Controller (TC), inside a tenant's domain, which dynamically manages virtual network functions for tenant's services. Next, it also includes the Element Management (EM), responsible for all the aspects/events related to virtual network functions such as configuration, performance, fault, security, etc. Second, the NFVI consists of an Infrastructure SDN Controller (IC), which sets up and manages network resources to guarantee available connectivity for intervirtual-network-function communications. It also logically hosts the physical resources and their mapping into virtual resources (through the virtualisation layer). Finally, the MANO hosts the Virtualized Infrastructure Manager (VIM), which controls and manages the NFVI resources and the IC. Next, the Virtual Network Function Manager (VNFM) configures and manages the life cycle of virtual network functions in its network domain. Finally, there is(are) also orchestrator(s) for NFVI resource management across different VIMs, and for managing the life cycle of network services.

This discussion has shown the growing virtualisation process, starting from the outsourcing of computing and widening to the virtualisation of routing, data and control plane, and of any network function. This process has been reaching an additional level of generalisation by also including the virtualisation of the protocol stack [1]. The idea behind Programmable Protocol Stack (PPS) is to realise a software-based environment for the adaptive management of protocols and protocol stack layers. This means that the system can reassign/change parameters, update services, and replace functionalities according to the needs of the users, network, and environment. This has become highly necessary due to the difficulty of satisfaction and prediction of QoS requirements in highly heterogeneous scenarios.

\section{THE ADVENT AND THE EVOLUTION OF 5G}

In 2016, 5G standardization effort started. In particular, the ITU Telecommunication Standardization Sector (ITU-T) Focus Group on IMT-2020 concluded its prestandardization activities in December 2016 and around the same time, ITU-R concluded its Recommendation "Vision of IMT beyond 2020" [16]. Side by side, in 2015, the New Generation Mobile Networks (NMGM) Alliance, consisting of Members (i.e. operators) with contributions from Sponsors (i.e. vendors) and Advisors (i.e. research entities) Partners, had already published its white paper/deliverable on the $5 \mathrm{G}$ vision [17]. The collaborative and global structure of this standardization process and the standardization bodies and the entities involved, have already been shown in Fig. 2. But, what was the key element that mainly stimulated the paradigm shift from LTE to $5 \mathrm{G}$ ?

It is now important to notice that the characteristics and performances achieved by LTE had made this wireless technology highly attractive for several verticals, apart from mobile broadband. However, LTE has inherent limits that cannot make it suitable for a wide range of heterogeneous verticals. This advancement very clearly emerges from the NMGM Alliance's deliverable statement "[...] The boundaries between personal and enterprise usage of devices will blur. [...]" [17], which clearly shows this change of paradigm between $1 \mathrm{G}-4 \mathrm{G}$ and $5 \mathrm{G}$. With $5 \mathrm{G}$, the service requested by the UE drives the goals of the communication network and the subsequent design of network architecture and characteristics.

\begin{tabular}{|c|c|}
\hline $\begin{array}{l}\text { Broadband access in dense areas } \\
\text { - Person-to-person or person-to- } \\
\text { group video communication } \\
\text { with extremely high resolution, } \\
\text { - Smart Office, }\end{array}$ & $\begin{array}{l}\text { Broadband access everywhere } \\
\text { - } 50+\text { Mbps Everywhere, } \\
\text { - Highly flexible network to be } \\
\text { deployed under ultra-low cost } \\
\text { requirements. }\end{array}$ \\
\hline $\begin{array}{l}\text { - Cloud services provided by } \\
\text { operators, } \\
\text { - HD Video/Photo Sharing in } \\
\text { Stadium/Open-Air Gathering. }\end{array}$ & \multirow{2}{*}{$\begin{array}{l}\text { Higher user mobility } \\
\text { - High Speed Train, } \\
\text { - Remote Computing, } \\
\text { - } \text { Moving Hot Spots, } \\
\text { Connectivity: Aircrafts, } \\
\text { balloonists, gliders, or } \\
\text { skydivers. } \\
\end{array}$} \\
\hline $\begin{array}{l}\text { Massive Internet of Things } \\
\text { - Smart Wearables, } \\
\text { - Sensor Networks, } \\
\text { - Mobile Video Surveillance. }\end{array}$ & \\
\hline $\begin{array}{l}\text { Extreme real time communications } \\
\text { - Tactile Internet. }\end{array}$ & \multirow{2}{*}{$\begin{array}{l}\text { Lifeline communications } \\
\text { - Public safety and emergency } \\
\text { services, } \\
\text { - Robust communications in case } \\
\text { of natural disasters. }\end{array}$} \\
\hline \multirow{3}{*}{$\begin{array}{l}\text { Ultra-reliable communications } \\
\text { - Automated Traffic Control and } \\
\text { Driving, } \\
\text { - Collaborative Robots, } \\
\text { - eHealth Extreme Life Critical, } \\
\text { - Remote Object Manipulation } \\
\text { (e.g. Remote Surgery), } \\
\text { - 3D Connectivity via Unmanned } \\
\text { - Aerial Vehicles, } \\
\text { - Public Safety. }\end{array}$} & \\
\hline & \begin{tabular}{|ll} 
Broadcast-like services \\
- & News and Information \\
- & Local, Regional and National \\
& Broadcast-like Services \\
\end{tabular} \\
\hline & \\
\hline
\end{tabular}

Fig. 4 - Groups of main use cases/verticals identified in [17]. 
In fact, the document from the NMGM Alliance [17] started drafting a possible initial classi ication of 5G use cases, which is depicted in Fig. 4. This also implied a preliminary and general definition of system's Key Performance Indicators (KPI) including a user's experienced data rate, end-to-end latency, mobility, connection density, traffic density, spectrum efficiency, coverage, resource and signalling efficiency. However, the authors of [18] had already mentioned some potential technologies, which could have been exploited to host the several potential-upcoming verticals. First, they envisioned the employment of massive Multiple-Input Multiple-Output (MIMO) and mmWave frequencies in the new RAN to boost the data rate. Next, they looked at the existing research, standardization, and implementation of cloud computing and network virtualisation (see Section 2.2) and suggested the pivotal role that softwarization and computing could have had in 5G. This is not only important for the core network but also for the evolution of the RAN towards a Cloud Radio Access Network (C-RAN) paradigm.

Afterwards, several verticals were finally organised into three main categories [19] according to their common KPI. First, enhanced Mobile Broadband (eMBB) including mobile broadband and mobile video streaming, with throughput and availability as main requirements. Next, ultra-reliable Machine-Type Communications (uMTC), or Ultra-Reliable Low-Latency Communication (URLLC), refers to communications whose requirements are concurrently and mainly focused on bandwidth, latency and reliability. Finally, massive Machine-Type Communication (mMTC) will support massive communications within IoT, eHealth sensors/wearables, smart grids and surveillance; its requirements are focused on bandwidth supply for a large number of devices and reliability.

Another major breakthrough in $5 \mathrm{G}$, compared to previous generations, is the change of the communication network paradigm [1]. Until 4G and in parallel within the Internet, information routed from the source(s) to the $\operatorname{sink}(\mathrm{s})$ was merely stored and queued, waiting for the availability of link resources to be transmitted. This paradigm is normally called store-and-forward. The softwarized architecture of $5 \mathrm{G}$ with the key role of network functions, running within softwarized environments (e.g. virtual machines, containers, etc.), has moved the focus on computing. In this sense, $5 \mathrm{G}$ represents the first communication network based on compute-and-forward.

\subsection{Performance and metrics}

We have seen that $5 \mathrm{G}$ is user-centred, so that the performance and design requirements are driven by the verticals. By looking at KPI, what marked an important change in $5 \mathrm{G}$ (in respect of previous generations) was not merely the definition of more stringent goals. The turning point was represented by their concurrent satisfaction, from which rose critical technological trade-offs. Various new services that $5 \mathrm{G}$ is planning to host require KPI which sometimes conflict with each other. In detail, the targeted values of the KPI for $5 \mathrm{G}$ networks have been proposed by the industry [19], [20] to be:

- throughput/data rate up to $1-10 \mathrm{Gbit} \mathrm{s}^{-1}$,

- end-to-end latency down to $1-10 \mathrm{~ms}$,

- 1000 times increase in bandwidth per unit of area,

- $99.999 \%$ perceived availability and 100\% geographical coverage,

- frame error rate ${ }^{1}$ equal to $1-10^{-5}$ [21],

- $90 \%$ reduction in network energy usage,

- significantly-increased battery life for various kinds of end-users' devices.

- localisation precision equal to $10 \mathrm{~cm}$ in two dimensions [21],

- spectrum efficiency three-five times greater than the one of $4 G$ [22],

- density of connected devices $10^{6} \mathrm{~km}^{-2}$ [22],

- receiver sensitivity about $-120 \mathrm{dBm}$ [22].

Those proposals have then been provided to the IMTprocess, leading to the technical performance requirements Report ITU-R M.2410 produced by ITU-R Working Party 5D (WP 5D), which is responsible for the overall radio system aspects of International Mobile Telecommunications (IMT) systems, comprising IMT-2000, IMTAdvanced, IMT-2020 and IMT for 2030 and beyond.

Even if it was important in previous generation networks, the frame error rate becomes critical in 5G since URLLC groups very sensitive services like remote surgery. In particular, the concept of frame error rate links with reliability, which is characterised by its reliability rate, that is "[...] defined as [...] the amount of sent packets successfully delivered to the destination within the time constraint required by the targeted service, divided by the total number of sent packets. Note that the reliability rate is evaluated only when the network is available. [...]" [17]. It is possible to see from this definition and from the previous list that network availability (i.e. a high availability rate) becomes subsequently critical and so, the related concept of resilience. In detail, resilience is defined as "[...] the capability of the network to recover from failures [...]" [17]. Additionally, the request is also that the network should be capable to somehow remotely self-heal.

An important consideration on the KPI also concerns latency, which highlights another big difference between $5 \mathrm{G}$ and the previous generations. While until LTE latency issues were mainly concerning the links within the RAN

\footnotetext{
${ }^{1}$ The frame is the data unit at Layer 2 of the International Organization for Standardization (ISO)-Open Systems Interconnection (OSI) proto-
} col stack. 
and the core network, with 5G, the latency switches to an end-to-end perspective. As previously mentioned, the $5 \mathrm{G}$ network has a softwarized architecture thus, the network functions can run on servers that belong not only to operators' computing hardware but also to data centres in the Internet, which are networks themselves. So, as a consequence, the evaluation of the KPI should be on an end-to-end basis. That is also the case of energy efficiency. Energy efficiency "[...] is defined as the number of bits that can be transmitted per Joule of energy, where the energy is computed over the whole network, including potentially legacy cellular technologies, Radio access and Core networks, and data centres. [...]" [17]. Given this, it appears clear that the virtualisation of the RAN can maybe improve its energy efficiency but, on the other hand, it can also reduce it within the core network or the data centres.

Next to KPI, another important metric of communication networks is quality. In particular, there can be three possible metrics to measure 'quality': QoS, Quality-ofPerception (QoP) (or user-perceived QoS), and Quality-ofExperience (QoE). Network QoS is the "[...] degree of conformance of the service delivered to a user by a provider with an agreement between them [...]" [23]. Next, the QoS is the "[...] totality of characteristics of a telecommunications service that bear on its ability to satisfy stated and implied needs of the user of the service [...]" [23]. The QoS relies on technical metrics so it is technology-centred. The QoP "[...] is primarily concerned with the detectability of a change in quality or the acceptability of a quality level. [...]" [23]. As an example, the use of Mean Opinion Score (MOS) measures the 'perception of quality' according to a subjective rating.

The target of $5 \mathrm{G}$ has also been the support for verticals, in which users are immersed as 'protagonists' (e.g. Tactile Internet, Augmented/Virtual Reality, etc.) [24]. This 'immersion' of human end users in the communication services has made the QoS an incomplete metric. Because of that, the concept of QoE appeared, which can be defined as QoE, or user-level or user-assessed QoS. The QoE relies on end-user behaviour/perception so, it is user-centred. However, the QoE is a harder metric to quantify since it involves subjective evaluation.

In the literature, two definitions of QoE can be found. The first states that QoE is a "[...] measure of user performance based on both objective and subjective psychological measures of using an ICT service or product. [...]" [23]. For this definition, ETSI particularly mentions two notes:

- "[...] It takes into account technical parameters (e.g. QoS) and usage context variables (e.g. communication task) and measures both the process and outcomes of communication (e.g. user effectiveness, efficiency, satisfaction and enjoyment). [...]" [23];
- " [...] The appropriate psychological measures will be dependent on the communication context. Objective psychological measures do not rely on the opinion of the user (e.g. task completion time measured in seconds, task accuracy measured in number of errors). Subjective psychological measures are based on the opinion of the user (e.g. perceived quality of medium, satisfaction with a service). [...]" [23].

Next, the second definition states that the QoE is the "[...] overall acceptability of an application or service, as perceived subjectively by the end-user [...]" [23]. It is important to notice that the QoE is an end-to-end metric, which can also be affected by users' expectations and context. According to the above definitions and considerations, 5G clearly arises more difficulties in the evaluation of communication performances. This is not only due to its end-to-end communication perspective and its heterogeneity (as an ecosystem of heterogeneous network architectures, communications, and services), but it is also due to the prominent and wide role of subjective metrics, which had very narrow and limited impact in previous generation networks and in the Internet.

\subsection{G architectural characteristics}

$5 \mathrm{G}$ has been the first communication network trying to inherently incorporate the network virtualisation paradigms, together with cloud computing. Moreover, 5G started combining SDN and NFV, following the guidelines of the ETSI MANO SDN-NFV architecture (see Fig. 3). In parallel, the concept of SDR was also included within 5G in order to have an end-to-end softwarized/virtual network, making the radio access technologies more flexible and reconfigurable. In this end-to-end virtual network context, the virtual network functions have become service functions, which process ingoing information at the various layers of the protocol stack. Next, service functions can be dynamically ordered into specific logical chains, according to the network tasks that have to be performed on communication messages. More precisely, a Service Function Chaining (SFC) is defined as "[...] an ordered set of abstract service functions and ordering constraints that must be applied to packets and/or frames and/or flows selected as a result of classification. [...]" [25].

The combination of all these technologies, that have been mentioned, has enabled the adaptive-optimal-flexible allocation of resources, but also the possibility for their logical isolation. This has opened the way for another communication network paradigm called end-to-end network slicing [1], [26]-[29]. Network slices are logical and 'isolated' virtual networks that can be instantiated on a unique physical network infrastructure. These virtual networks are generated by the allocation of subsets of available physical resources and by the set of policies to identify the specific traffic flows, that are going to be hosted. In fact, slices can be created to manage and operate multi-tenant communication scenarios or to address 
the satisfaction of KPI of a set of verticals. In particular, end users can also belong to more than a slice, and slices can also be defined for limited subsets of the network in-stead of on an end-to-end basis.

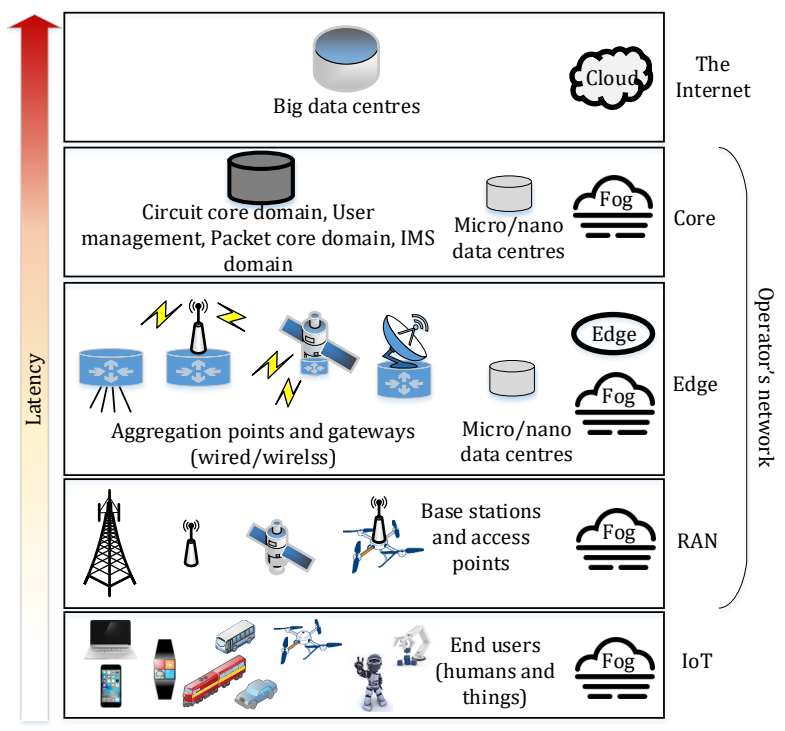

Fig. 5 - Logical high-level structure of 5G, specifying the location of fog, cloud, and edge computing.

Fig. 5 depicts the high-level general end-to-end architecture defined for $5 \mathrm{G}$, graphically clarifying some characteristics and definitions. As briefly described in Section 2.1, the RAN consists of access points (non-3GPP access technologies) and base stations. Next, base stations, or more precisely eNodeBs, consist of two main elements: the Remote Radio Head (RRH), or Remote Radio Unit (RRU), and the Baseband Unit (BBU), which are interconnected via a fibre optical link using the Common Public Radio Interface (CPRI) protocol. The aim of the BBU is to perform the processing due to the lower layers of the wireless cellular network protocol stack. The BBU also deals with Forward Error Correction (FEC), Medium Access Control (MAC), and encryption/decryption of Packet Data Convergence Protocol (PDCP) uplink/downlink communications.

Next, the front haul is defined as "[...] The intra-base station transport, in which a part of the base station function is moved to the remote antenna site. [...]" [14]. On the other hand, the back haul is defined as "[...] The network path connecting the base station site and the network controller or gateway site. [...]" [14]. These two definitions are important to be taken into account once discussing how $5 \mathrm{G}$ includes the virtualisation paradigm of $\mathrm{C}$ RAN. By employing and implementing C-RAN, the BBU is softwarized and so, it runs in a virtual environment (for example, in a virtual machine or a container), which is normally placed in the cloud. This means that the tasks of each layer of the BBU, mentioned in the previous paragraphs, have to seamlessly be performed in a cloud data centre. However, by looking at Fig. 5, this implies a significant increase in latency. If we assume the values re- ported in [30], [31], the link between the RRU and the cloud server (also considering the delay caused by the in-troduction of a virtual environment) would have to satisfy a latency of $150 \mu$ s at a rate of $2457 \mathrm{Mbit} \mathrm{s}^{-1}$. This would be the case of softwarizing tasks until the very lower lay-ers. Instead, if we target the softwarization of just the up-per layers, the so-called Split D, including FEC and Hybrid Automatic Repeat Request (HARQ), the requirements re-lax to $150 \mu \mathrm{s}$ at a rate of $180 \mathrm{Mbit} \mathrm{s}^{-1}$ (latencies around $1 \mathrm{~ms}$ can be accepted at the price of an increased error rate, experienced by UEs).

These considerations about C-RAN can be similar for other services requiring very low latency. That is why, the concepts of fog and edge computing in 5G have emerged. The definition of edge is more articulated and less clear than the other ones. Specifically, the idea of edge and Mobile Edge Computing (MEC) derived from the original concept of fog computing, which arose as a more distributed solution to target URLLC extending the centralised vision (with increased latency) provided by cloud computing. Between 2011 and 2012, the term fog computing was created within Cisco Systems Inc. and it was defined as "[...] a highly virtualized platform that provides compute, storage, and networking services between end devices and traditional Cloud Computing Data Centers, typically, but not exclusively located at the edge of network. [...]" [32]. This definition actually means that the concept of fog computing, more general than that of MEC [33], [34], means that computing can be distributed anywhere in be-tween the cloud and the end users/things, as correctly un-derlined by [35].

Given these premises and the characteristics of $5 \mathrm{G}$, it is important to define a system providing multi-access MEC, that is a network "[...] which provides an IT service environment and cloud-computing capabilities at the edge of an access network which contains one or more type of access technology, and in close proximity to its users. [...]" [36]. So, what can we name as 'edge'? First, it is possible to say that the edge of the network depends on the specific vertical that is targeted. Next, if we consider the virtualisation of BBU functionalities within edge micro/nano data centres, the initially-clear definition of RAN overlaps with the edge, and it can still be accurate to say that the RAN becomes a subset of the edge. Thus, the clear separation, graphically shown in Fig. 5, would become more shaded. Recently, there has been a harmonisation process between different standardization efforts by ETSI and 3GPP regarding MEC [37], towards the design of a common unique architecture for $5 \mathrm{G}$ MEC.

After the high-level architectural discussion above, it is important to show some details about $5 \mathrm{G}$ architecture as expressed in the latest published 3GPP release [38]. By now, $5 \mathrm{G}$ New Radio (NR) has been the principal standardization effort. This has the scope of providing an efficient and effective access network in order to connect different 


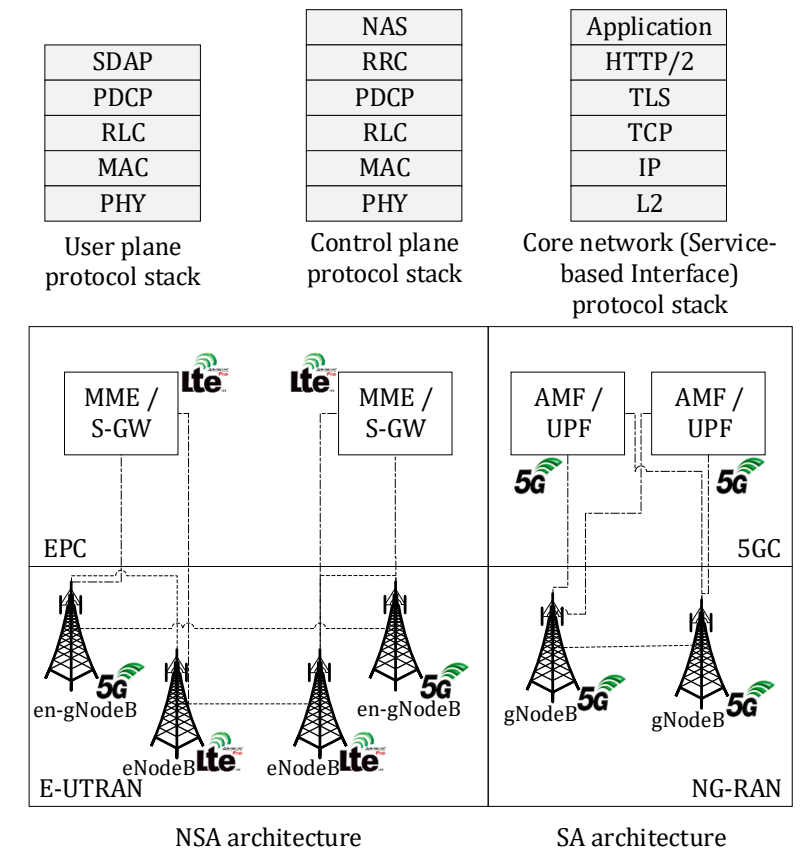

Fig. 6 - 5G architectural characteristics and proposed protocol stacks according to [38].

types of UEs' services/verticals. 5G RAN will not only offer connectivity to a $5 \mathrm{G}$ core network and users, so-called Stand-Alone (SA) architecture, but it will also support the interconnection of LTE UEs and core network, the socalled Non-Stand-Alone (NSA) architecture (see Fig. 6). The NSA architecture is the intermediate approach that will be employed until 5G NR will have completely been deployed. This RAN consists of $5 \mathrm{G}$ base stations (logical en-gNodeB) and legacy LTE eNodeBs so that the connectivity to UEs happens via 5G NR and E-UTRAN. In this scenario, the eNodeB is the master node and the en-gNodeB is the secondary node. On the other hand, the SA architecture represents the full $5 \mathrm{G}$ deployment, once LTE will not operate anymore. This will imply a RAN only consisting of gNodeBs. The detailed frequency bands of 5G NR are listed in Fig. 7.

Next, the 5G Core Network (CN) architecture is designed to ensure strong support to the critical virtualisation paradigms that we have previously analysed, such as MEC and network slicing. Fig. 6 depicts in detail what has been defined by 3 GPP until now. In contrast with EPC, the $5 \mathrm{G}$ $\mathrm{CN}$ (or 5GC) employs virtualisation so that it becomes a Service-Based Architecture (SBA) [38], where the architecture elements are defined as network functions instead of network entities. The necessary network functions that have been defined are the User Plane Function (UPF) (dealing with users' data), the Application Function (AF) (handling the applications), the Access and Mobility Management Function (AMF) (accessing the UE and the RAN), and the Session Management Function (SMF) (accessing the UPF).
5G NR standard sets three main protocol stacks: core network (service-base interface), user plane, and control plane protocol stacks. In the services provided by $5 \mathrm{G} \mathrm{CN}$, network functions are designed as a set of Application Programming Interface (API), relying on the core network (service-base interface) protocol stack depicted in Fig. 6 (top-right). The employed interface definition language is OpenAPI 3.0.0. This protocol stack employs the serialisation protocol JavaScript Object Notation (JSON) ${ }^{2}$ (IETF RFC 8259), the application layer protocol HTTP/2 (IETF RFC 7540), the Transport Layer Security (TLS) protection, the Transmission Control Protocol (TCP) (IETF RFC 793), and the IP.

Next, the control plane protocol stack starts with the physical layer, which performs the modulation/demodulation of the signal on the radio interface. The MAC sublayer deals with tasks such as mapping between logical and transport channels, scheduling, and error correction via HARQ. Next, the Radio Link Control (RLC) sublayer mainly transfers the upper layer Protocol Data Unit (PDU), and performs sequence numbering independent of the one in PDCP and error correction through Automatic Repeat reQuest (ARQ). The PDCP sublayer principally performs sequence numbering, header compression/decompression, transfer of users' data. The Radio Resource Control (RRC) establishes, configures, maintains and releases Signalling Radio Bearers and Data Radio Bearer (DRB); it also manages mobility, handovers, and cell selection for UEs, while also handling QoS functions. Finally, the Non-Access Stratum (NAS) manages all the aspects and protocols just transported by the access network to the $5 \mathrm{G} \mathrm{CN}$ : in fact, it ends in the AMF. Finally, the user plane protocol stack is similar to the control plane protocol stack except for the presence of the Service Data Adaptation Protocol (SDPA) layer rather than the RRC and NAS. The SDPA layer maps between radio bearers into different QoS flows and marks each QoS flow with a specific ID in both downlink and uplink.

\section{THE CONCEPT AND VISIONS OF 6G}

Given the global success that $5 \mathrm{G}$ has had, ITU has started writing the report entitled 'IMT Future Technology Trends Towards 2030 and Beyond', which is going to be released in June 2022. This report will be the input for the ITU recommendation 'Vision of IMT beyond 2030', which will give the very general vision and guidelines for the global network of networks that $6 \mathrm{G}$ will be. As a starting point, external organizations have been invited to contribute to the new ITU-R Report and new ITU-R Recommendation [39]. Because of that, in the last few years, speculations have been proposed in order to give some shape to the future 6G networks. After the imprint left by $5 \mathrm{G}$, even $6 \mathrm{G}$ started from the definition of potential targeted use cases.

\footnotetext{
${ }^{2}$ The serialisation protocol converts complex objects to sequences of bits.
} 
Among the various proposed verticals, Augmented Reality (AR) and Virtual Reality (VR) have been the main and most popular drivers of $6 \mathrm{G}$ vision. Even if already proposed in a preliminary form during $5 \mathrm{G}$, now AR and VR visions have significantly been extended towards the realisation of 3D holographic video representations and interactions. Some initial studies have estimated that "[...] a raw hologram, without any compression, with colors, full parallax, and $30 \mathrm{fps}$, would require $4.32 \mathrm{Tbit} \mathrm{s}^{-1}$. The latency requirement will hit sub-millisecond, and thousands of synchronized view angles will be necessary [...]" [40]. Additionally, the idea has also been to make holo-grams physically perceptible by associating not only au-diovisual information but also haptic data as in the Tactile Internet.

These initial speculations have also brought to the potential realisation within communication networks of a more advanced concept, called digital twin. A Digital Twin [41] can be defined as a virtual representation of either physical objects, workflows, or generally speaking systems. The great initial interest has grown into the industrial community driving business specifically within the IoT [42]. In the context of the Industry 4.0, the design and production of specific products (e.g. bicycles, cars, etc.) can be enhanced and better managed via the interaction with the products' digital twin. A large number of sensors have to be applied on the real object to represent the physical characteristics into the virtual reality. This can help to find faults and to solve problems before the real-product creation. The concept of digital twin has also been extended from nonliving to living physical entities such as humans [42], [43]. According to what has just been said, it clearly appears that the quality of the virtual model, and of the big data collected and processed are pivotal [44], [45].

Some aspects have already been listed in the literature to start characterising the idea of digital twin [43]. First, all the digital twins will have a unique identifier. Sensors and actuators will be fundamental to guarantee complete and correct replication of the real twin into the virtual reality. These sensors will convey all possible kinds of data, not only audiovisual but also haptic, hearing, taste, smell, etc. Next, communication will also be critical. Digital and physical twin will have to experience real-time sensing and interactions. The phenomenology of the Digital Twin can assume different forms such as virtual (i.e. a 3D avatar), holographic, physical-robotic (e.g. a humanoid social robot), or software-based (i.e. via a set of software components without a recognisable form). Finally, trust (real twins must trust their digital counterparts) and privacy and security (a huge quantity of sensitive data is collected and processed) are fundamental pillars in the realisation and employment of digital twins. In this whole context, the role of Artificial Intelligence (AI) will be critical under many different angles. AI will be used to manage the communication network infrastructure, to process/act on data, to enforce security, etc. Thus, the paradigm of digital twins will open significant challenges to Machine Learning (ML) algorithms.

As just briefly mentioned above, multiple contexts of applications of digital twins have been identified. The first use case was industrial manufacturing [46]. Next, various other ones appeared such as remote surgery [47], construction industry [48], aviation [49] and aeronautics [50], oil and gas industry [45], [51], fitness [49]. From the standardization perspectives, the authors in [52] proposed the digital twins' standardization effort as the initial convergence from standards ISO/IEEE 11073, including X73 compliant devices.

As previously mentioned in Section 3.1, 5G verticals have been grouped into the three main categories eMBB, uMTC, and $\mathrm{mMTC}$. In the context of $6 \mathrm{G}$, these groups may respectively evolve to the ubiquitous Mobile Ultra-Broadband (uMUB), ultra-High-Speed Low-latency Communications (uHSLLC), and ultra-High Data Density (uHDD) [53]. Another consideration was proposed by [54], which suggested to include a new hybrid category of services called Mobile-Broadband Reliable Low-latency Communication (MBRLLC). In particular, this group of verticals includes services that jointly target the KPI of eMBB and uMTC applications. Side by side, it became the definition of massive URLLC [54], which jointly addressed verticals both targeting the KPI of mMTC and uMTC. This can imply an important conceptual observation. Until 4G-LTE, there was mainly a single mobile-broadband vertical. With $5 \mathrm{G}$, three distinct categories have been defined. In the context of $6 \mathrm{G}$, the taxonomy of verticals is going to become more and more elaborated, requiring more complex sets of KPI. This means the categorisation of services is going to show intersections among the groups.

\subsection{Envisioned performance and metrics}

As previously mentioned in Section 3.1, 5G has made requirements on the KPI more and more stringent, by also adding their concurrent satisfaction on a end-to-end perspective. According to the use cases that $6 \mathrm{G}$ vision has been targeting like the ones mentioned above, the preliminary list of requirements that has been set includes [21], [55], [56]:

- throughput/data rate up to $1 \mathrm{Tbit} \mathrm{s}^{-1}$,

- user-experienced data rate of $1 \mathrm{Gbits}^{-1}$ (ten times the one targeted by $5 \mathrm{G}$ ),

- end-to-end latency less than $1 \mathrm{~ms}$,

- an 'over-the-air' latency of $10-100 \mu$ s with mobility up to $1000 \mathrm{~km} \mathrm{~h}^{-1}$

- very broad bandwidth with frequencies reaching $1-3 \mathrm{THz}$, 
- "always-ON" terrestrial-aerial-satellite network,

- frame error rate (reliability) equal to $1-10^{-9}$ [21],

- very high energy efficiency also supporting "batteryfree IoT devices" (10-100 times the one of 5G [56]) and especially equal to $1 \mathrm{pJ} \mathrm{bit}^{-1}[21]$,

- connected intelligence,

- jitter equal to $1 \mu \mathrm{s}$ [21],

- spectrum efficiency greater than three times the one of $5 G$ [22],

- receiver sensitivity less than $-130 \mathrm{dBm}[22]$.

- a connectivity density ten times the one provided by $5 \mathrm{G}$, with an area traffic capacity of up to $1 \mathrm{Gbit} \mathrm{s}^{-1} \mathrm{~m}^{-2}\left(10 \mathrm{Gbit} \mathrm{s}^{-1}\right.$ in 3D [21]),

- density of connected devices greater than $10^{6} \mathrm{~km}^{-2}$ [22],

- localisation precision equal to $1 \mathrm{~cm}$ in three dimensions [21].

Moreover, in the very recent literature on 6G, this concept of KPI has been considered to be incomplete. Because of that, KPI and Key Value Indicators (KVI) have been placed side by side [2], [57]. The term KVI is based on the concept of value, which is defined as "[...] intangible yet important human and societal needs such as sustainability, trustworthiness, and inclusion. [...]" [2]. The KVI are grouped into three main categories [57]: growth, sustainability, and efficiency. The first is principally related to economic growth, and the creation of new values, business ecosystems and models. The second and the third mainly refers to digital inclusion, zero energy devices, resource efficiency and users' privacy. Additionally to the ones introduced for $5 \mathrm{G}$ in Section 3.1, other definitions of concept of quality have been introduced. The concept of Quality-of-Physical-Experience (QoPE) [54] is an attempt to complete and to unify the evaluation separately given by QoS and QoE, by combining them with other physical aspects of humans such as brain cognition, body characteristics, and gestures.

ITU has not yet started discussing requirements for "systems beyond IMT-2020". As part of the to be applied IMTprocess, ITU-R will produce a Report on "Technical performance requirements" (e.g. like ITU-R M.2410 for IMT2020) at a later stage.

\subsection{Targeted architectural characteristics}

By looking at the architectural characteristics of $5 \mathrm{G}$ and the current respective trends, everybody in the scientific and industrial community argues that 6G architecture will be completely softwarized and flexible. The current convergence between ETSI and 3GPP on this (previously cited for MEC) will extend to the full network architecture. In this sense, 6G will finally complete the paradigm shift from store-and-forward to compute-andforward [1], [58] or, more precisely, 6G will bring a "holistic management of communication" (including computing, caching, and control resources) as defined in [21].

The vision of $6 \mathrm{G}$ has also been enhancing the idea of 'ecosystem' of networks (or network of networks), preliminarily started with 5G. This has been making 6G closer and closer to the concept of the 'Web of Everything Everywhere' [59]. In fact, the research community agrees that there will finally be a full integration and interoperation between satellite, aerial and terrestrial network, merged in a unique dynamic-adaptive network infrastructure [60]-[62]. Moreover, some researchers have also envisioned the possibility of integrating underwater communication networks into the whole $6 \mathrm{G}$ network in order to provide seamless connectivity from the ocean/sea floor to the space [56]. Regarding the air to ground link, authors of [62] showed the link budget for the employment of $71-76 \mathrm{GHz}$ and $81-86 \mathrm{GHz}$ in the the air-to-ground link. This so-called 3D networking will significantly affect the design of 6G Layer 3/Layer 4 new network and transport protocols, which can be capable to efficiently and effectively exploit the three-dimensional characteristics of the communication networks [54]. Furthermore, the 3D network architecture has embraced the actual operators' network since, for example, it has been defined the concept of 3D core network [22]. The HighAltitude Platforms (HAP) and the low cost of nanosatellites' constellations will represent a pivotal means to enhance and ensure the complete and reliable connectivity to the rural areas [63].

As previously mentioned D2D communications were initially introduced in LTE networks. Next, 5G vision significantly stressed the employment of D2D technologies (together with mobile small cells [64]) in order to allow for the provision of effective connectivity in dense scenarios. Nowadays, the D2D paradigm has also been included in the 6G vision [65]. The ultra-dense scenarios envisioned in $6 \mathrm{G}$, if we also consider the complexity added by 'hybrid verticals' such as MBRLLC and massive URLLC, have been identified as important justifications for a massive and seamless integration of D2D communications and mobile small cells (possibly cooperative) into the future $6 \mathrm{G}$ ecosystem of networks.

6G communication networks will be the first generation of networks with native AI. This means that AI will not merely be an application but an inherent part of the infrastructure, and of the network management and operations [66]. The usage of AI for physical, network, and application layers was described in [67]. While for network and application layers the ideas come from existing research in ML and Self-Organising Networks (SON)/autonomic networking, the additional novel aspect is the full application of AI within the physical layer. The idea is to make intelligent operations such as channel 
modelling and estimation, spectrum management, power control, handover management, etc. Next, traffic and mobility prediction, together with supervised ML management of policies were additionally mentioned by [68], in the context of URLLC. The authors in [69] envisioned an intelligent protocol stack, in which AI handles analysis, management, and optimisation of the operations per layer. The employment of AI for the physical layer's operations was also highlighted by [70]. Instead of focusing on a protocol-stack perspective, the authors of [70] discussed the usage of AI from the RAN perspective. The study and design of an intelligent RAN for $6 \mathrm{G}$ will impact not only on the performance, management, and operations but also on the design and future standardization of RAN internal devices and technologies [70]. Next, [71] dealt with the design of an intelligent edge.

Each generation of wireless cellular networks has been promising an increase in data rate. In order to do that, a combination of augmenting spectral efficiency and bandwidth should be employed. This is something that $6 \mathrm{G}$ has also been promising [58]. The former can be obtained by going on employing massive MIMO antennas; the latter by going towards higher and higher frequencies. Next, wireless communications using THz frequencies are the solution to achieve Tbit $^{-1}$ data rates. The pro of these frequencies is the provisioning of very narrow beams, which can eventually mitigate interference and help in augmenting the possible number of antennas into the base stations [56]. Fig. 7 clearly shows the 5G NR operating bands together with the targeted 6G RAN operating band [72]. The table in the figure also specifies what bands are assigned for the Time-Division Duplex (TDD), the Frequency-Division Duplex (FDD), the Supplementary Uplink (SUL), and the Supplementary Downlink (SDL).

The vision of $6 \mathrm{G}$ has also pictured the realisation of a wireless 6G RAN flexibly using time-frequency-space resources [73]. Regarding frequency, it has been envisioned the usage of mmWave and Terahertz bands, and likely visible-light band. By referring to time, 6G could target a subsequent reduction of the duration of the time slot in order to better serve very low-latency verticals. In the space domain, it could further enhance the trend of $5 \mathrm{G}$, by employing base stations equipped with ultramassive MIMO technologies. Base stations transmitting within the Terahertz band will guarantee a coverage of about $5 \mathrm{~m}$ to guarantee a User Datagram Protocol (UDP) data rate of 1 Tbits $^{-1}$ [72]. For distances greater than $7-10 \mathrm{~m}$ mmWaves will be able to achieve greater data rates [72]. Wireless links so directive and so short will need the design of new medium access techniques in order to efficiently exploit the new bandwidths, as underlined in [72]. On the other hand, these characteristics can make Terahertz frequencies interesting as backhaul links [72]. Highly important is also the design of new antennas with specific geometries and physical characteristics in order to efficiently provision the connectivity and the targeted data rates [74]. For example, some initial channel models for future 6G communication networks have been presented in [75].

The authors of [76] proposed some key physical-layer problems to be solved to move from $5 \mathrm{G}$ to $6 \mathrm{G}$ physical layer. In particular, $6 \mathrm{G}$ should improve $5 \mathrm{G}$ via the exploitation of higher frequency bands (mmWaves and especially Terahertz), of smart radio technologies such as Reconfigurable Intelligent Surface (RIS), and the full realisation of a cell-less massive MIMO wireless system. The main characteristic of cell-free or cell-less systems is that the 'usual' concept of cell is abandoned and access points and base stations in the RAN coherently serve end users, using the same time-frequency resources in a specific covered area. This can imply the elimination of cells' boundary effects [76].

As shown in Fig. 7, the imagined 6G RAN has also been targeting the exploitation of frequencies in the visible-light spectrum.

Since the beginning of $6 \mathrm{G}$ research, Visible Light Communications (VLC) have been considered by industry and academia as an excellent candidate complementary technology to provide optical fibre-like connectivity performance [21], [77]. The use of visible light spectrum with VLC offers the potential to create short-range (less than $10 \mathrm{~m}$ ) high-capacity links with ultrahigh bandwidth (Terahertz), and zero electromagnetic interference with radio frequencies. Today available VLC products have limited performance from few tens of $\mathrm{Mbit} \mathrm{s}^{-1}$ over short ranges (up to $5 \mathrm{~m}$ ). As explained in [21], at the horizon of 2024, upcoming new light sources based on micro-LED technology will unlock such limitations, enabling the use of $1 \mathrm{GHz}$ bandwidth (and more), and achieving tens of Gbit s ${ }^{-1}$ with single-diode LEDs (even up to several hundreds of Gbit s$^{-1}$, thanks to the coming availability of micro-LED matrices and dedicated optical beamforming algorithms allowing for spatial separation of users). Next, in a few years' time (by 2027), it is expected that by adding massive parallelisation of micro-LED arrays and dedicated wavelength division multiplexing techniques, VLC will be able to offer, similarly to sub-THz communications, to target Tbit s $^{-1}$ aggregated throughput (see Fig. 8).

Further above, we have alluded to RIS employment within 6G. A first step into a radical change, that has been offered to future 6G networks, is the inclusion of an RIS concept within the wireless network architecture. 5G communication networks have been following the Shannon's communication paradigm, which establishes the principles for the reliable transmission of symbols over a noisy communication channel [78]. However, current wireless communications extend their role from pure communication systems to much more complex ones, involving the interaction between natural and artificial intelligence, responding to multifold requirements, and being able to exploit revolutionary techniques to control various 


\begin{tabular}{|c|c|c|c|c|}
\hline \multirow{2}{*}{$\begin{array}{l}\text { DAB/FM radio } \\
\text { Television } \\
300 \mathrm{MHz} \\
450 \mathrm{MHz} \\
\end{array}$} & $\begin{array}{c}\text { NR } \\
\text { band }\end{array}$ & Uplink (UL) & Downlink (DL) & $\begin{array}{l}\text { Duplex } \\
\text { Mode }\end{array}$ \\
\hline & $\mathrm{n} 1$ & $1920 \mathrm{MHz}-1980 \mathrm{MHz}$ & $2110 \mathrm{MHz}-2170 \mathrm{MHz}$ & FDD \\
\hline \multirow{4}{*}{$\begin{array}{c}\text { Frequency } \\
\text { Range } 1 \\
\text { (5G and LTE) }\end{array}$} & $\mathrm{n} 2$ & $1850 \mathrm{MHz}-1910 \mathrm{MHz}$ & $1930 \mathrm{MHz}-1990 \mathrm{MHz}$ & FDD \\
\hline & n3 & $1710 \mathrm{MHz}-1785 \mathrm{MHz}$ & $1805 \mathrm{MHz}-1880 \mathrm{MHz}$ & FDD \\
\hline & n5 & $824 \mathrm{MHz}-849 \mathrm{MHz}$ & $869 \mathrm{MHz}-894 \mathrm{MHz}$ & FDD \\
\hline & n7 & $2500 \mathrm{MHz}-2570 \mathrm{MHz}$ & $2620 \mathrm{MHz}-2690 \mathrm{MHz}$ & FDD \\
\hline $7125 \mathrm{MHz}$ & n8 & $880 \mathrm{MHz}-915 \mathrm{MHz}$ & $925 \mathrm{MHz}-960 \mathrm{MHz}$ & FDD \\
\hline \multirow[b]{6}{*}{$24250 \mathrm{MHz}$} & $\mathrm{n} 12$ & $699 \mathrm{MHz}-716 \mathrm{MHz}$ & $729 \mathrm{MHz}-746 \mathrm{MHz}$ & FDD \\
\hline & n20 & $832 \mathrm{MHz}-862 \mathrm{MHz}$ & $791 \mathrm{MHz}-821 \mathrm{MHz}$ & FDD \\
\hline & $\mathrm{n} 25$ & $1850 \mathrm{MHz}-1915 \mathrm{MHz}$ & $1930 \mathrm{MHz}-1995 \mathrm{MHz}$ & FDD \\
\hline & n28 & $703 \mathrm{MHz}-748 \mathrm{MHz}$ & $758 \mathrm{MHz}-803 \mathrm{MHz}$ & FDD \\
\hline & n34 & $2010 \mathrm{MHz}-2025 \mathrm{MHz}$ & $2010 \mathrm{MHz}-2025 \mathrm{MHz}$ & TDD \\
\hline & n38 & $2570 \mathrm{MHz}-2620 \mathrm{MHz}$ & $2570 \mathrm{MHz}-2620 \mathrm{MHz}$ & TDD \\
\hline \multirow{6}{*}{$\begin{array}{c}\text { Frequency } \\
\text { Range } 2 \\
\text { (5G mmWaves) }\end{array}$} & n39 & $1880 \mathrm{MHz}-1920 \mathrm{MHz}$ & $1880 \mathrm{MHz}-1920 \mathrm{MHz}$ & TDD \\
\hline & $\mathrm{n} 40$ & $2300 \mathrm{MHz}-2400 \mathrm{MHz}$ & $2300 \mathrm{MHz}-2400 \mathrm{MHz}$ & TDD \\
\hline & $\mathrm{n} 41$ & $2496 \mathrm{MHz}-2690 \mathrm{MHz}$ & $2496 \mathrm{MHz}-2690 \mathrm{MHz}$ & TDD \\
\hline & n50 & $1432 \mathrm{MHz}-1517 \mathrm{MHz}$ & $1432 \mathrm{MHz}-1517 \mathrm{MHz}$ & TDD1 \\
\hline & n51 & $1427 \mathrm{MHz}-1432 \mathrm{MHz}$ & $1427 \mathrm{MHz}-1432 \mathrm{MHz}$ & TDD \\
\hline & $\mathrm{n} 66$ & $1710 \mathrm{MHz}-1780 \mathrm{MHz}$ & $2110 \mathrm{MHz}-2200 \mathrm{MHz}$ & FDD \\
\hline \multirow{6}{*}{$52600 \mathrm{MHz}$} & n70 & $1695 \mathrm{MHz}-1710 \mathrm{MHz}$ & $1995 \mathrm{MHz}-2020 \mathrm{MHz}$ & FDD \\
\hline & n71 & $663 \mathrm{MHz}-698 \mathrm{MHz}$ & $617 \mathrm{MHz}-652 \mathrm{MHz}$ & FDD \\
\hline & n74 & $1427 \mathrm{MHz}-1470 \mathrm{MHz}$ & $1475 \mathrm{MHz}-1518 \mathrm{MHz}$ & FDD \\
\hline & n75 & $\mathrm{N} / \mathrm{A}$ & $1432 \mathrm{MHz}-1517 \mathrm{MHz}$ & SDL \\
\hline & n76 & $\mathrm{N} / \mathrm{A}$ & $1427 \mathrm{MHz}-1432 \mathrm{MHz}$ & SDL \\
\hline & n77 & $3300 \mathrm{MHz}-4200 \mathrm{MHz}$ & $3300 \mathrm{MHz}-4200 \mathrm{MHz}$ & TDD \\
\hline \multirow[t]{2}{*}{$300000 \mathrm{MHz}$} & n78 & $3300 \mathrm{MHz}-3800 \mathrm{MHz}$ & $3300 \mathrm{MHz}-3800 \mathrm{MHz}$ & TDD \\
\hline & n79 & $4400 \mathrm{MHz}-5000 \mathrm{MHz}$ & $4400 \mathrm{MHz}-5000 \mathrm{MHz}$ & TDD \\
\hline \multirow{4}{*}{$\begin{array}{l}\text { 6G Proposed } \\
\text { Frequency } \\
\text { Range } \\
\text { (Terahertz) }\end{array}$} & n80 & $1710 \mathrm{MHz}-1785 \mathrm{MHz}$ & $\mathrm{N} / \mathrm{A}$ & SUL \\
\hline & n81 & $880 \mathrm{MHz}-915 \mathrm{MHz}$ & $\mathrm{N} / \mathrm{A}$ & SUL \\
\hline & n82 & $832 \mathrm{MHz}-862 \mathrm{MHz}$ & $\mathrm{N} / \mathrm{A}$ & SUL \\
\hline & n83 & $703 \mathrm{MHz}-748 \mathrm{MHz}$ & $\mathrm{N} / \mathrm{A}$ & SUL \\
\hline \multirow{2}{*}{$\begin{array}{r}3000000 \mathrm{MHz} \\
30000000 \mathrm{MHz}\end{array}$} & n84 & $1920 \mathrm{MHz}-1980 \mathrm{MHz}$ & $\mathrm{N} / \mathrm{A}$ & SUL \\
\hline & n86 & $1710 \mathrm{MHz}-1780 \mathrm{MHz}$ & $\mathrm{N} / \mathrm{A}$ & SUL \\
\hline $\begin{array}{l}\text { IR } \\
400000000 \mathrm{MHz}\end{array}$ & n257 & $26500 \mathrm{MHz}-29500 \mathrm{MHz}$ & $26500 \mathrm{MHz}-29500 \mathrm{MHz}$ & TDD \\
\hline \multirow{2}{*}{$\begin{array}{l}\text { Visible Light } \\
790000000 \mathrm{MHz}\end{array}$} & $\mathrm{n} 258$ & $24250 \mathrm{MHz}-27500 \mathrm{MHz}$ & $24250 \mathrm{MHz}-27500 \mathrm{MHz}$ & TDD \\
\hline & $\mathrm{n} 260$ & $37000 \mathrm{MHz}-40000 \mathrm{MHz}$ & $37000 \mathrm{MHz}-40000 \mathrm{MHz}$ & TDD \\
\hline UV & n261 & $27500 \mathrm{MHz}-28350 \mathrm{MHz}$ & $27500 \mathrm{MHz}-28350 \mathrm{MHz}$ & TDD \\
\hline
\end{tabular}

Fig. 7 - NR bands specified in the 3GPP 5G Rel-15 [38] (subsequently included in 3GPP TS 38.104 Rel-17 of June 2021) and specification of the Terahertz and visible light frequency bands that $6 \mathrm{G}$ will target. 


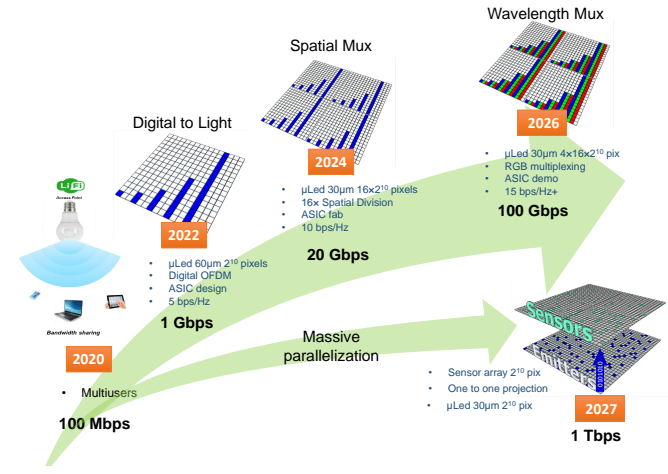

Fig. 8 - Visible light communication road map from Mbit $\mathrm{s}^{-1}$ to Tbit $\mathrm{s}^{-1}$. Figure courtesy of [21].

aspects of the communication-computation-control chain [79]. Following the Shannon's mantra, the common assumption in wireless communications has been that the channel is given and it cannot be altered according to the communication needs. However, with the advent of RIS, there is the possibility to adjust the communication channel to control wireless connectivity and mitigate interference. In this way, it is possible, for example, to increase the channel capacity without necessarily increasing neither the transmit power nor the bandwidth, or to reduce the associated electromagnetic field footprint. Radical technological advances based on the emerging paradigm of RIS [80] are offering today the opportunity to forge a new generation of dynamically-programmable wireless propagation environments with minimal redesign and reconfiguration costs for the connect-compute network.

RIS can act especially as a transmitter, receiver or as an anomalous reflector, where the direction of the reflected wave is no longer specular according to natural reflection laws, but instead adaptively controllable. This can offer unprecedented opportunities to locally support a dynamic adaptation to stringent and highly-varying 6G service requirements such as momentary link capacity, localisation accuracy, energy efficiency, electromagnetic field emission and secrecy guarantee. By definition, RIS are artificial intelligent controlled surfaces, constituted by hundreds or thousands of reconfigurable unit elements. They can be embedded in parts of the environment, such as walls, mirrors, ceilings, etc. and can operate as a nearlypassive tunable anomalous reflector or as a transmitter/receiver, when equipped with active radio-frequency elements. Nowadays, RIS operate at low frequency but research is actively designing a solution to support wideband operations up to the sub-THz spectrum. In particular, RIS can be implemented using a variety of technologies and, through its property of modifying the radio wave propagation, can provide extraordinary benefits for diverse wireless goal-oriented communications. Different antennas' technologies can be adopted to design RIS, along with reflect-arrays [81], transmit-arrays [82], [83] and, smart, programmable or software defined metasurfaces [84] [85].
Although RIS have great potentials to implement advanced electromagnetic wave manipulations, several fundamental and implementation problems are still unsolved. At the physical layer, only simple functionalities, such as electronic beam-steering and multibeam scattering, have been demonstrated in the literature. In addition, problems such as channel state information estimation and acquisition, passive information transfer and transceiver design are still open. At the network layer, the propagation settings of installed RIS might be adapted depending on scenarios, application needs and on realtime/predicted network dynamics. As today, open challenges remain on how to define the network architecture incorporating multiple RIS and how to orchestrate the reconfiguration of multiple RIS devices in time-space, to meet specific suitable (goal-oriented) deployment strategies for effectively exploiting RIS technology. Such RIS network adaptation capabilities should enable dynamic programming of the wireless propagation environment while meeting specific legislation and regulation requirements on spectrum use and electromagnetic field emission, that might vary for specific locations and evolve over time. Finally, it is still an open question to check under what conditions RIS-empowered networks can provide a significant reduction of the overall network energy usage.

\section{WHAT SHOULD 6G BE?}

Previously, Section 2 and Section 3 discussed wireless cellular networks and 5G, while Section 4 generally described the technological, architectural, and metricrelated trends envisioned for 6G. These important aspects were aimed at providing the fundamental background for now trying to answer the questions, which were originally stated in the Introduction: What can $6 G$ be? Do we really need 6G? In the last few years some articles [86], [87] have already tried to pose and to initially address these questions, showing some concerns regarding the need for a new 'network generation' after 5G. So, we now address various critical points in order to justify how $6 \mathrm{G}$ can really differentiate from $5 \mathrm{G}$. Then, the following general analysis will help justifying the need for the so-called new generation, underlining how $6 \mathrm{G}$ is not merely the answer to the disregarded promises of $5 \mathrm{G}$, but it is a new disruptive generation of communication networks. The following will assume the perspective of KPI and metrics and the one of new verticals (and subsequent needed technologies), which were the main drivers for the advent of $5 \mathrm{G}$ and have also been the main forces for the rise of $6 \mathrm{G}$.

\subsection{Performance indicators and metrics}

Let us now discuss the current set of performance indicators and metrics for 6G (see the list in Section 4.1) to see if they are really motivated or if they just represent a needless increase of the ones of $5 \mathrm{G}$. The first criticism refers to the KPI linked to higher bandwidth and data rate. We agree with [86], which correctly states that increasing 
data rates to 1 Tbit s$^{-1}$ is something that end users will surely welcome but not at an augmented price, since it is not a prominent need. An increase in data rate without $6 \mathrm{G}$ would still be possible, since, as discussed in [87], 5G has already foreseen the inclusion of higher frequencies up to $60 \mathrm{GHz}$. Thus, covering higher frequencies would not necessarily require the huge additional investments for a new 6 th generation in order to get additional very broad bandwidth with frequencies reaching $1-3 \mathrm{THz}$. Furthermore, the targeted data rate of $5 \mathrm{G}$ set to $1-10 \mathrm{Gbit} \mathrm{s}^{-1}$ can be enough for the satisfaction of many possible verticals already envisioned or to come. For example, the initial requirements estimated for 3D holographic communications [40], and implicitly for the digital twin, of 4.32 Tbit s $^{-1}$ are for raw data. This quantity could significantly be reduced with advanced data compression techniques that could be researched. This means that more efficient and effective methodologies to compress data could avoid the usage of unnecessary spectrum and unnecessary investments by the operators. In this direction, recent works have been exploring the potential of Semantic Communications [88] to achieve maximum compression of data while ensuring the correct accomplishment of identified tasks between interacting entities.

Moreover, since the advent of $4 \mathrm{G}$ services, network traffic has been drifting from mainly downlink to uplink intense usage, because of various new bandwidth-intensive applications [89]. Representative examples of services driving to such a paradigm shift in cellular networks are video sharing, real-time MEC offloading support, cloud backup, massive IoT data gathering, etc. With $5 \mathrm{G}$ we are already experiencing such dramatic inversion of the direction of traffic. With 6G, many of the new types of services will even push the network usage to higher imbalances, with much more uplink traffic in many scenarios and use cases.

Each UE connected to the network implies a specific amount of processing load required at the BBU. The processing required by a $\mathrm{UE}$ for the uplink in $4 \mathrm{G}$ was expressed in [90] as

$$
p_{U E}=\left(3 A+A^{2}+\frac{1}{3} M C L\right) \frac{R}{10}
$$

where $A$ is the number of antennas, $M$ the modulation bits, $C$ the code rate, $L$ the number of spatial MIMO-layers and $R$ the number of Physical Resource Block (PRB). The processing load $p_{U E}$ is measured in Giga-Operations Per Second (GOPS). Even if defined for $4 \mathrm{G}$, Eq. ((1)) can give us an idea of the impact that requirements and trends of 6G previously mentioned can have on computing, latency, and energy usage. A data rate in the order of Tbit $\mathrm{s}^{-1}$ increases the value of $R$ as the number of MIMO antennas that $6 \mathrm{G}$ is planning to use augments $L$. For example, in [73], it is envisioned the employment of $1024 \times 1024$ MIMO elements. This means that the data rate will highly increase computing, which will increase the overall latency and energy usage. This concern underlines the need for compression techniques, which can avoid the need for the envisioned transmission rates.

Regarding latency, end-to-end latency consists of the sum of various contributions [91], [92]. The propagation latency is the physical distance between communication entities. This is physically bounded by the speed of light and can only be reduced via techniques that somehow 'virtually' shorten the real communication distance such as MEC. Next, transmission latency depends on the inverse of the available capacity on the communication link. The queuing latency comes from the scheduling of data transmissions (e.g. prioritisation) and routing. In a network ecosystem, where softwarization makes in-network computing the pillar of any network functionality, the processing is a key aspect. Thus, the processing latency is the delay, which depends on the hardware's processing capacity of network nodes.

By considering that $6 \mathrm{G}$ will be a fully-intelligent network, another latency variable jumps into the calculation of end-to-end latency. We may call it intelligence latency. By considering the complete deployment and integration of $\mathrm{AI}$ into the communication network management and operations, it is important to notice that a new paradigm arises, also bringing with it its own cons: big data. In fact, intelligence requires continuous big data collection, preprocessing and analysis performed in a distributed manner by various data centres within the whole network. This means an explosion of the control traffic, which can become comparable to the amount of data traffic sent across the network. This means that a $6 \mathrm{G}$ intelligent control plane [93] will require time not only for distributed data mining and classification but also for training, deciding, and acting on the network environment (we do not consider now the delay for the network reconfiguration after the intelligent algorithm has acted). In this context, the use of proactive ML algorithms may help to make latency ultra-low [94] or 'negative' [95]. This paradigm is the so-called 'anticipatory networking'. The two terms of this paradigm [96] include anticipation, the exploitation of prediction techniques or the assumption of given future knowledge, and networking, the optimisation of mobile communications. However, anticipatory networking is somehow incompatible with targeting null failure because prediction (and so ML algorithms) is not deterministic and it has a variable accuracy (with probability always less than 1), whose quality also strictly depends on the data previously collected.

Finally, softwarization implies the deployment of virtual machines and containers. It is well-known that the complete and massive use of software is not as efficient as the hardware-based solutions [1], [97]. This means that the open challenge of matching $5 \mathrm{G}$ latency requirements with softwarization will be physically quite hard, especially when targeting the extreme range of values less than $1 \mathrm{~ms}$ with 6G. Additionally, the explosion of the data rate to 1 Tbit s $^{-1}$ will probably make impossible the re- 
duction of transmission, queuing, and processing latency, for example, if we take into account the virtualisation of signal processing in the context of C-RAN and BBU splitting.

Another critical aspect among 6G KPI is energy efficiency. The very high data rate, the massive application of virtualisation, native AI with continuous data mining and processing (so-called connected intelligence) will increase in-network computing to an unprecedented level. Next, an 'always $0 N^{\prime}$ ' $6 \mathrm{G}$ network (i.e. experiencing no failures) will require the presence of backup virtual network functions, always ready for a replacement. Moreover, a large density of UEs, together with several of them needing Tbit $^{-1}$ data rate, will greatly augment the computing required per user at the BBU. Especially, this will be a virtual BBU according to the C-RAN paradigm. Thus, this huge stress on edge and cloud data centres will significantly increase the end-to-end energy usage. Next, in order to improve power efficiency for coverage and battery life, the network operating region will have to enlarge in both the regions of the power and spectral efficiency [58]. As also mentioned in [58], it will be hard for 6G to concurrently enhance coverage, cost, and battery life. We also agree with [58] that higher data rates and efficiency will make the possibility of increasing battery life more difficult. All these observations about energy underline some inconsistencies among the KPI, that will be extremely hard to solve by just evolving the current 'classic' technologies. $6 \mathrm{G}$ could be a quite successful story for energy usage just by creating a full intelligent autonomic network while satisfying the KPI originally established for $5 \mathrm{G}$ (in fact, this energy target has not been reached yet).

In contradiction with the reduction of energy usage advertised within $6 \mathrm{G}$, there is also the technological trend of mobile small cells and fog computing at the UEs, for very low latency. Mobile small cells integrate the concept of mobile relay stations with fixed small cells and can be deployed into vehicles such as public transport systems [98]. In [99], authors showed that mobile cells can improve spectrum efficiency, throughput, and signal quality of users. Future communication is expected to be a network of a large number of mobile users that will interact together with many applications such as VR/AR games or streaming videos requiring low response times. Therefore, mobile small cells can be deployed via cluster of UEs using D2D technology [100], [101]. This type of small cell can also be deployed to cover the urban landscape. These cells can be formed using mobile UEs or low-cost nodes such as RRHs which are connected to aggregation nodes through wireless links [64]. The base stations of these cells mainly perform a transceiver's functionalities and are less involved than fully functional eNodeBs. In the literature, mobile small cells are also called on-demand because they can flexibly be deployed in case of significant traffic variations in short-time ranges, etc. All these considerations show that battery life will necessarily in- crease to optimise the coverage and the usage of spectrum in very dense urban scenarios, and also it will augment to support fog computing within the IoT network layer (see Fig. 5).

According to what has been seen in Section 4, we can assume that $6 \mathrm{G}$ will see an increased importance of QoE versus QoS, compared to what has been for $5 \mathrm{G}$. This is due to the increased focus on users' experience quality and satisfaction, together with the prominent immersion of users into the VR. Even in the 'natural' reality, the users are expected to seamlessly interact with virtual entities, the socalled AR. AR will especially become more and more extreme starting from 3D holographic images, towards objects and avatars. These holograms will change the way we work, we experience reality, and more generally will create 'cross-reality' users (either humans or machines), acting in a 'mixed' or 'extended' [102] reality between VR and the 'natural' reality. In this context, the advancements of the research in neuroscience and psychology will be pivotal for the success of these technologies in communications and 6G, and for the measurement of their quality. Given these considerations, we can also agree with the introduction of hybrid metrics combining QoS and QoE as the previously mentioned QoPE (see Section 5.1).

On the other hand, other metrics such as KVI [2], [57] may create confusion and not be really effective in evaluating performance and in providing useful design guidelines. As previously quoted, the definition of value indicator refers to an 'intangible human and societal need'. However, in the moment of design and measurement, an intangible need is always mapped into either objective (e.g. QoS) or subjective (e.g. QoE) metrics, or into a combination of both (e.g. QoPE). For example, the same can happen for the mentioned value 'sustainability', which can be defined as "[...] the long-term viability of a community, set of social institutions, or societal practice. In general, sustainability is understood as a form of inter-generational ethics in which the environmental and economic actions taken by present persons do not diminish the opportunities of future persons to enjoy similar levels of wealth, utility, or welfare [...]" [103]. From this definition, it appears clear that, in order to measure and to target 'sustainability' within 6G design and development, it has to be mapped into its economic, societal, and environmental KPI.

After having critically discussed potential $6 \mathrm{G}$ use cases and performance metrics, the following deals with $6 \mathrm{G}$ technological and architectural aspects.

\subsection{Network virtualisation and campus net- works in 6G}

By looking at Section 3, it is possible to see the distance between the promises referred to $5 \mathrm{G}$ network virtualisation and the actual virtual-architectural characteristics, provided by the current standardization and develop- 
ment. Then, $6 \mathrm{G}$ will need to complete the process of network softwarization that has already started with $5 \mathrm{G}$, not only with the full deployment of the ETSI MANO architecture but also considering the existing MEC harmonisation between ETSI and 3GPP, that we have previously mentioned. Additionally, 6G should finalise the softwarization process by applying the paradigm of PPS [1] to the whole networks' infrastructure protocol stack.

Another important aspect regarding virtualisation is the evolution of the control plane. $6 \mathrm{G}$ should go beyond the design of a distributed control plane, leveraging the current strong trend on micro-services and multi-agent systems. This will also help for the seamless integration between the virtualised infrastructure and the native AI. In this sense, 6G's current design and future subsequent standardization will not have to neglect the important ongoing standardization and design efforts by ONF within the $\mu$ ONOS project. This will add quite higher flexibility and adaptability to the control plane, while also creating the fertile ground for the full exploitation of the potentials of native AI.

In the next stages of the development of $5 \mathrm{G}$ networks, the 5 G RAN will have to guarantee the provisioning of connectivity to the entire population with mobile data communications at any time and in any place, and the concurrent service provisioning for industrial full automation. Initial 5G campus networks have already been set up for industry, municipalities or educational institutions. These networks differ in their coverage, as they are designed to serve only one factory or villages. The further development of campus networks and their full integration into the whole network infrastructure should become a priority of 6G. In addition to pure cost reduction, they can also guarantee security, lower energy usage and further flexible software solutions for the RAN, such as the so-called OpenRAN solutions. OpenRAN is an open interface down to the antenna that can be reconfigured at any time by software.

The original definition of campus network (also called specialised network) states that it represents a network of various Local Area Network (LAN) within a limited geographical area. In this sense, 6G will further generalise this original concept, by defining campus networks as networks of LANs, with their access networks and edge computing resources. The full employment of future campus networks will be a pivotal RAN-edge paradigm of the network infrastructure under 6G. They will mainly support mobility within their domain without necessarily interconnect to other external networks. For example, 6G campus solutions can be deployed by network operators to provide customised and effective solutions to their industrial customers. This will allow $6 \mathrm{G}$ operators to ensuring the quality and the performances of the specific local campus networks that are deployed. Side by side, small operators can also benefit from campus solutions. For exam-
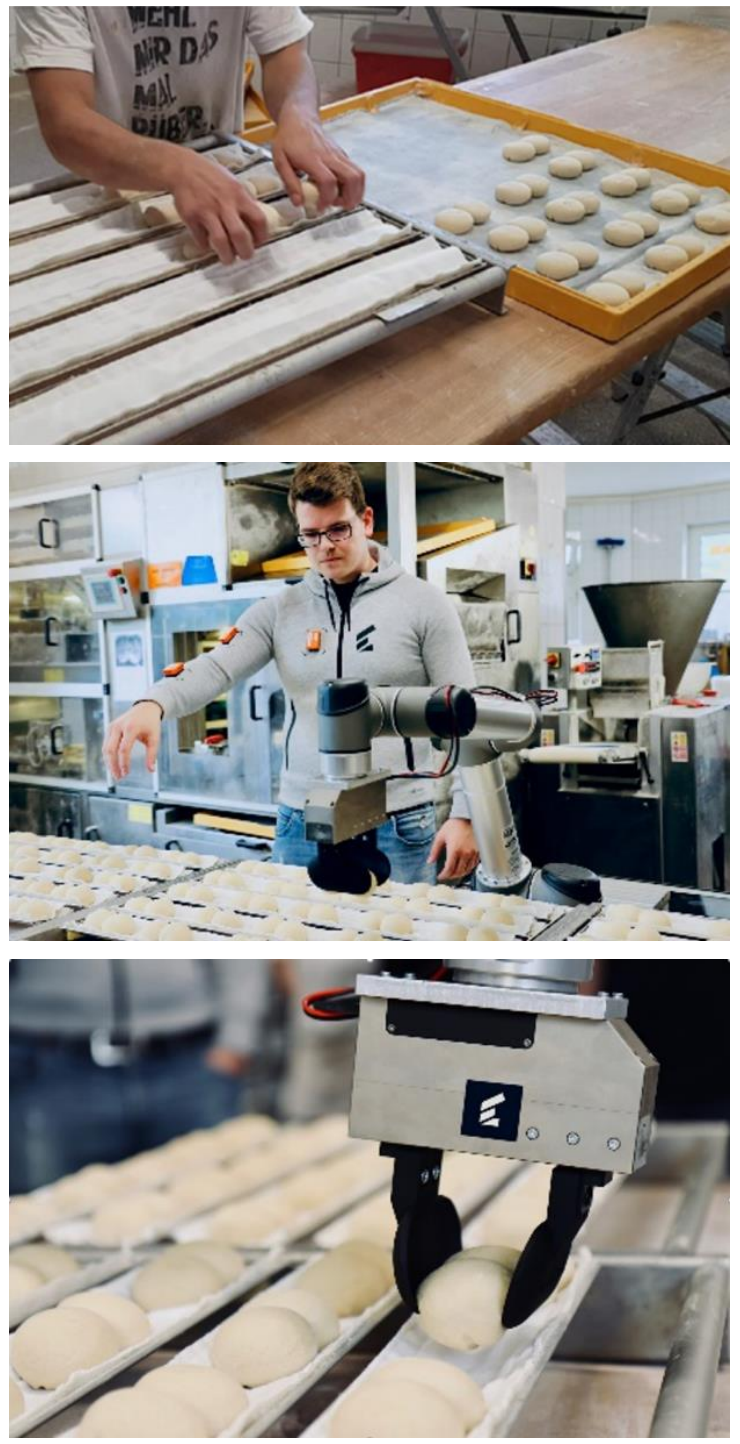

Fig. 9 - Application of a campus network for teaching a robot in a bakery (Copyright Wandelbots - free to use).

ple, a $6 \mathrm{G}$ campus can cover an entire region where smaller companies train robots such as bakeries (see Fig. 9).

Furthermore, the so-called paradigm of Industry X.0 [102] will massively exploit robots' automation and collaboration, among themselves and with humans. Hundreds of robots, sensors, and other hardware are expected to be all interconnected in a reliable way in 6G, also part of them requiring URLLC. Moreover, the continuous exploitation of human-related, machine-related, and network-related data will require low-latency flexible, secure, and dedicated edge computing. This scenario will represent the main motivation and the test bench for the evolution of campus networks within 6G architecture. The testbed in Fig. 9 shows some elements that highlight the need for 6G campus networks. With the higher and higher complexity of operations that robots are going to perform, the number of sensors that humans will wear is 
going to greatly increase. In fact, the increasing number of sensors allows for finer and more precise assisted operations. Next, these heterogeneous sensors will also imply continuous heterogeneous data collection and transmission to a dedicated intelligent edge within the campus network. Intelligence will not only be applied for network management and operations but also for robots training, acting, and deciding in order to better work autonomously or to assist humans. Thus, 6G campus networks will represent specialised and local networks of networks, providing high edge computing resources for such massive data handling.

\subsection{The tactile internet and digital twins}

The tactile Internet and haptic communications in general have been important drivers of $5 \mathrm{G}$ in the context of URLLC [104]. While today's Internet has been democratising information sharing, the tactile Internet has been targeting the democratisation of accessing skills and expertise, in order to promote equal possibilities for people of different ages, cultural backgrounds, geographical provenience, or physical limitations. In this context, the term 'tactile' describes the transmission of haptic information (related to touch and other senses) in order to actually feel and touch things in the virtual reality. Until now, only audiovisual information has been transmitted on the Internet and on wireless communication networks. The advent of the tactile Internet will dramatically change the way we learn (e.g., how to play the piano or various sports), how we work, or how we are assisted by machines as we age (see Fig. 10). This new form of collaboration between humans and machines also underscores the fact that digitisation will help economic and social growth, which have been important political requirements for 6G. Fig. 10 recalls the concept previously expressed about the need for $6 \mathrm{G}$ campus networks. A massive employment of sensors will involve various aspects and activities of human life. This will not only be limited to industrial activities but it will embrace more and more complex human operations like sports, remote medical assistance and surgery, etc. The conceptual depiction of Fig. 10 gives an idea of what massive and ubiquitous availability of the tactile Internet will imply. 6G unspecialised public networks will face unprecedented data communications, storage, and processing of heterogeneous data, considering low-latency requirements. These contexts cannot be supported by $5 \mathrm{G}$ technologies and their mere evolution.

While $5 \mathrm{G}$ has mainly been addressing communication between machines, whereas $6 \mathrm{G}$ will look more closely at the cooperation between humans and machines. Technically, the Tactile Internet with Human-in-the-Loop (TaHiL) [105] considers communication between the Master Domain, usually humans, and the Controlled Domain, which consists of machines. The peculiar characteristic of TaHiL communication is the fact of being a two-way communication (i.e. with feedback), which multiplexes different
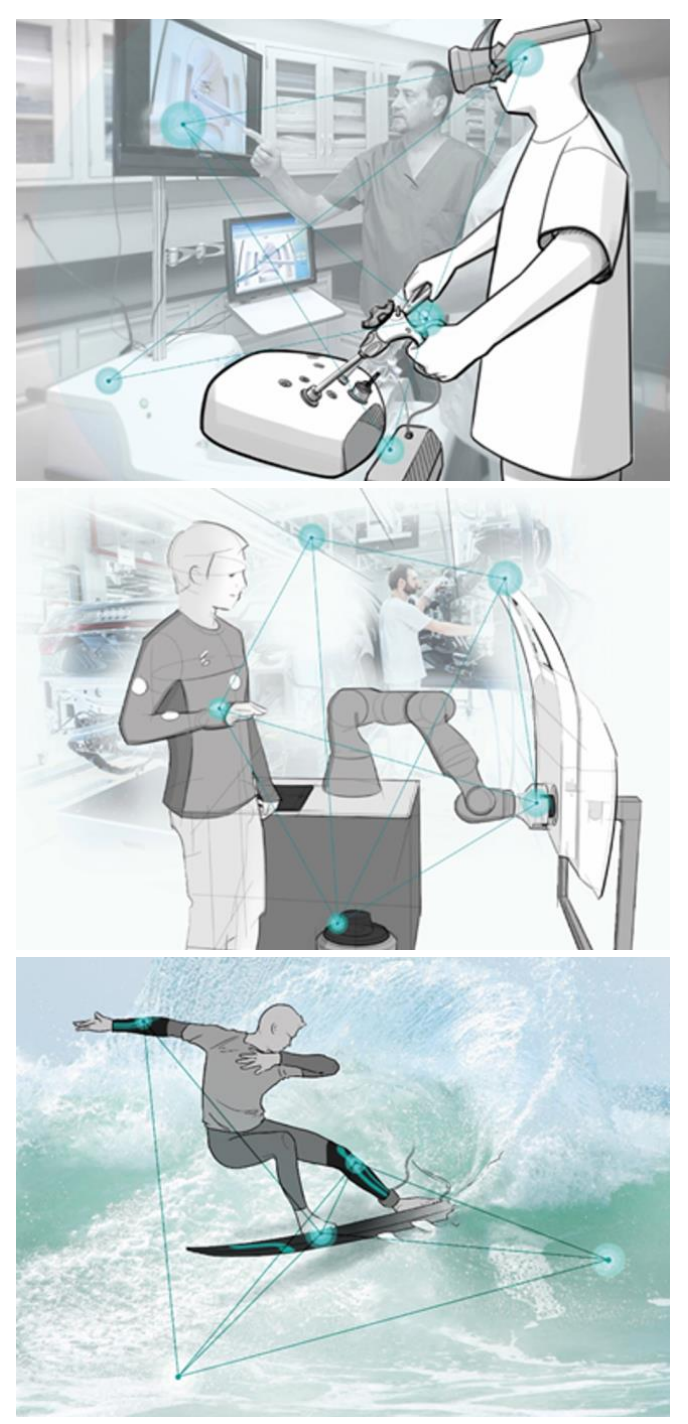

Fig. 10 - Vision of the Tactile Internet: Learning in medicine, industry and sports (Copyright: CeTI, TU Dresden - free to use).

kinds of data streams (audio, video and haptic), with different KPI to be concurrently satisfied. In fact, not only the input from the master should be transmitted to the controlled device, but also the input from the controlled entity should be rapidly and reliably sent back to the master. This allows the master for the successful and correct perception of the remote action, which has been performed. In fact, the scope behind the design is to make the communication between the so-called tactile edges seamless, without sensing the negative contributions of the intermediate-communication Network domain (latency, losses, etc.).

In 6G, the TaHiL will complete the shift from contentoriented to control-oriented communication. This aspect is still very far from current $5 \mathrm{G}$ standardization status, so it will be hard to see it clearly in the next releases. That is why, the TaHiL with the full realisation of end-to-end URLLC will see its zenith within the $6 \mathrm{G}$ era, and thanks to 
$6 \mathrm{G}$ network infrastructure. On the other hand, the technical design and development of TaHiL under 6G infrastructure will also be a test bench for the technologies and paradigms that are pillars for the realisation of digital twins. As previously explained in Section 4, digital twins will have several characteristics in common with general haptic communications and, specifically, with the TaHiL. In fact, digital twins will require an always-ON feedback loop between the virtual and real twins [43]. Moreover, they will empower and extend the haptic data used within the TaHiL to more general universal kind of data, which can fully convey and project the 'natural' reality of entities into the virtual reality.

\subsection{The unification of terrestrial, aerial, and satellite networks}

Next, 6G will also have to finally satisfy the promises regarding the seamless integration of terrestrial, aerial, and space networks. This promise somehow started during LTE [11], went on during $5 \mathrm{G}$, and now it has been forwarded to 6G (see the 6G KPI in Section 4). We believe that, for $6 \mathrm{G}$, now can be the perfect convergence of factors and circumstances to make this 'dream' coming true. The maturity, low cost and easiness to launch and deploy satellite platforms (e.g. the case of nanosatellites), and the amount of interest and investments on space, together with the real need of reliable/seamless coverage and lower latency, can be the perfect match to realise this promise in 6G.

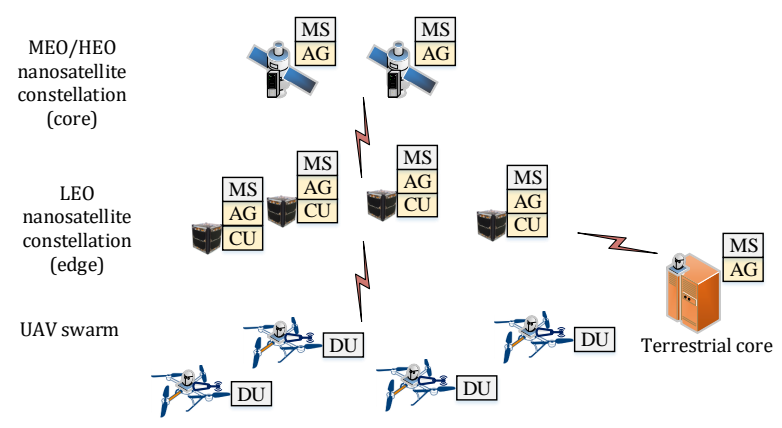

Fig. 11 - 6G three-dimensional architecture for coverage of rural and remote areas. Swarms of UAVs represent the base stations/access points hosting the Distributed Units (DUs) with the physical layer operations. Low Earth Orbit (LEO) constellations of nanosatellites represent the edge, in which Centralised Units (CUs) are hosted. Moreover, this LEO edge can also host micro-services (MS) and agents (AG), which perform any softwarized functionality or service. The yellow colour indicate that the service/function is intelligent. The core network can either be implemented in Medium Earth Orbit (MEO) or High Earth Orbit (HEO), or can regularly be hosted in terrestrial networks

In the case of using HAP, Unmanned Aerial Vehicles (UAV), and nanosatellites as edge network devices, it is important to choose bands that satisfy the KPI while also not interfering with existing terrestrial technologies. For example, authors in [106] have shown the need for $2400-2483.5 \mathrm{MHz}$ and $2300-2450 \mathrm{MHz}$ bands for the satellite-based C-RAN. However, these choices are suitable for connectivity in remote regions (which is the use case in [106]) but in various areas these bands may interfere with terrestrial IEEE 802.11-based, industrial, scientific, and medical services.

Future 6G networks aims to go beyond the enhancement of terrestrial services, with the support of non-terrestrial networks. With 6G a new ambition has arisen. New multidimensional mobility services will enable end users and machines moving in the three-dimensional (3D) space, being able to access (on demand) to connectivity and intelligence support. Therefore with 6G, KPI on localisation precision and uniform user experience will be specifically defined for terrestrial services and for services in the 3D space [60]. Fig. 11 depicts an example of a 6G 3D scenario to provide coverage in rural and remote areas. The UAV swarms perform operations limited to the physical layer, the so-called Distributed Unit (DU), in order to maximise the battery lifetime and operations. This means that constellations of Low Earth Orbit (LEO) nanosatellites will be deployed to host the MEC, providing computing resources for the virtualised RAN and the Centralised Unit (CU). According to what has been previously mentioned regarding anticipatory networking, the 6G CU is going to be intelligent. In general, intelligent agents will be hosted in the nanosatellites to perform functions for lower-latency verticals. Next, the core network will be both terrestrial and orbital. Medium Earth Orbit (MEO) and High Earth Orbit (HEO) satellites will realise distributed orbital data centres, providing computing for micro-services and intelligent agents requiring higher computational resources. A big open challenge will be the establishment, control, and maintenance of micro-service/agents chains in such complex network infrastructure. Moreover, the realisation of distributed data centres in satellite constellations for $6 \mathrm{G}$ 3D networks will also require significant investigation. In fact, satellites have power and time constraints (they use solar power and provide coverage for a limited amount of time).

\subsection{The role of intelligence in $6 \mathrm{G}$}

Next, as already mentioned above while discussing latency, intelligence - AI and ML - for 6G can be the same as softwarization and computing for 5G. As written in Section 2.2, the original research and standardization efforts of Internet Engineering Task Force (IETF) and ETSI were absorbed and merged into the vision and standardization of $5 \mathrm{G}$ wireless cellular networks, which had subsequently started. The work of the ETSI Industry Specification Group (ISG) Experiential Networked Intelligence (ENI) started around 2017 with the publication of the White Paper [107]. The main scope of ETSI ISG ENI is to design an intelligent architecture for network management, taking into account context-aware policies to adjust network service provisioning according to users' needs, environmental conditions, and business goals. 


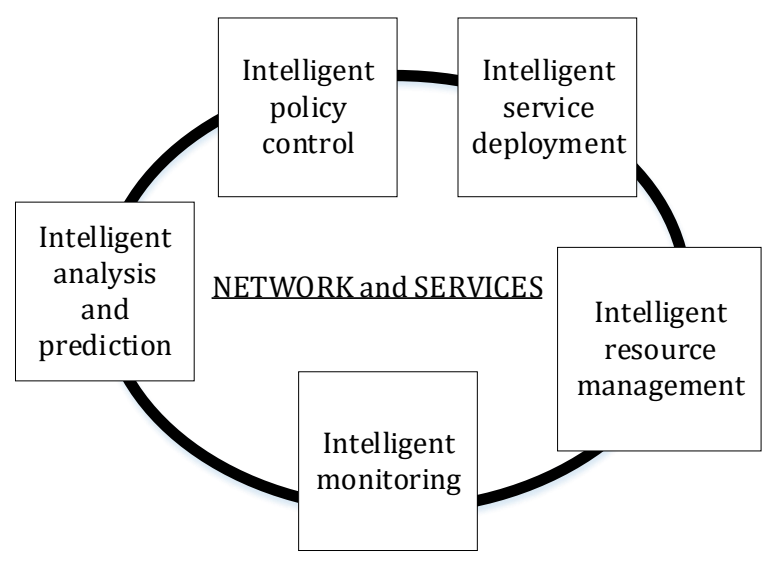

Service and Network Requirements

- General requirements

- Service orchestration and management

- Network planning and deployment

- Network optimisation

- Resilience and reliability

- Security and privacy

Functional Requirements

- Data collection and analysis

- Policy management

- Data learning

- Interworking with other systems

- Mode of operations

- Model training and iterative optimisation

- API requirements

Non-Functional Requirements

- Performance requirements

- Operational requirements

- Regulatory requirements

Fig. 12 - Circle of functionalities of the ETSI ENI system. Groups and list of ETSI ENI requirements regarding how intelligence is applied to the network and applications to improve network operations, management and experience of service provisioning.

The scope of the ETSI ENI framework is to continuously capture network system's configurations in order to taking actions to dynamically change the system's characteristics, according to the targeted objectives and KPI. As already mentioned before, while we were discussing the latency issues of 6G, ETSI ENI intelligent architecture relies on big data mining and analysis, which are input for ML to train, learn, decide, and act. According to these aspects, ETSI ENI can automate complex network humandependent decision-making processes to increase network performances. Fig. 12 depicts the intelligent functionalities of the ETSI ENI architecture. By considering the pure architectural characteristics, the ETSI ENI system considers the heterogeneity of the existing and future hardware network infrastructure together with the full virtualisation obtained via the ETSI MANO SDN-NFV architecture. On top of that, there is the AI of ENI, which interacts with and manages the specific assisted systems distributed across the network. This is made possible via a specific API [107], [108].

The recent document [109] defines a clear list of the requirements for the ETSI ISG ENI architecture. These requirements are important to evaluate and control how AI works within the network and the applications to improve network operations, management and service provisioning. Fig. 12 also shows these requirements and how they are grouped. Finally, the use cases that have been identified for the ETSI ENI system can be categorised into five main groups [110]: infrastructure management, network operations, service orchestration and management, assurance, and network security. The first considers the processes related to the management of the network infrastructure such as maintenance, planning, and allocation of services. The second concerns with the operations of the network so, the extraction and analysis of the runtime contexts and the optimisation of management operations. The third handles the orchestration and management of orders and services, taking into account specific different Service Level Agreement (SLA) of verticals. The fourth deals with network monitoring and prediction of future network states in order to ensure optimal maintenance and continuous service delivery. Finally, the deployment of AI also targets network security.

Side by side, ETSI has also been investigating a reference model for autonomic networking, cognitive networking and self-management of networks and services. This specific architecture is called ETSI Generic Autonomic Networking Architecture (GANA), published in 2016 [111]. This architecture is directly inspired by the idea of SON, nevertheless it provides a more general reference which is able to interoperate with complementary technologies such as SDN, NFV, and big data analytics for Autonomic Management and Control (AMC). The idea of $\mathrm{AMC}$ relies on the definition of Decision-making-Element (DE), which is an autonomic function that is a cognitive control-loop in centralised/distributed management and control planes. The DE owns self-* features such as selfconfiguration, self-optimisation, self-healing, etc. Each DE is an adaptive entity, which dynamically monitor and manage its respective management entity. Practically, a DE is placed within a network node at a specific layer of the protocol stack. Additionally, each DE can be either a real or a softwarized entity.

This brief excursus on ETSI ENI and GANA has been important to underline the concept that the complete integration of AI into 6G cannot neglect the experience provided by ETSI in the last five-six years. A significant part of research on intelligence in $6 \mathrm{G}$ has not even been mentioned in this research. For example, the only article dealing with AI and 6G, cited in Section 4, just briefly mentioning ETSI ENI is [69]. None of the others even cite either ETSI ENI or GANA. However, ETSI ENI and GANA architectures will be pivotal for $6 \mathrm{G}$ standardization as ETSI MANO SDN-NFV architecture has been fundamental for 5G. 
Regarding intelligence and 6G, there are some critical challenges that have to be addressed in order to realise the vision of intelligent automation. We agree with [69] that issues related to training, correct evaluation of performance and set up of a lower bound for the AI KPI are hard goals because of both the complexity of a communication network ecosystem like $6 \mathrm{G}$ and the lack of explicability of AI processes and results. Moreover, we also agree that various ML models and algorithms are hard to be generalised and maybe the future solution we can envision will be an intelligent multi-agent system, where multiple ML algorithms collaborate. On the other hand, we may disagree with [69] regarding the problem of interoperability since, if $6 \mathrm{G}$ will fully realise the complete softwarization of the network and the massive deployment of general purpose hardware (started with 5G), the coexistence of various AI modules will be effectively guaranteed by coexistence and 'social' collaboration of various intelligent network agents.

\subsection{Beyond Shannon with semantic communi- cations}

Until the design of $5 \mathrm{G}$ networks, communication has been the basic commodity of every wireless generation. The key challenge has been the reduction of the uncertainty associated to the correct reception of exchanged data, while targeting higher capacity, reliability, and lower latency. Such legacy of Shannon's model has pushed a never-stopping race for broader bandwidths, thus exploring higher frequency bands. Today, kicking-off research on future 6G networks, the need for a paradigm shift from mainstream research, which builds on Shannon's information theory, starts taking shape. With 5G, the communication network has evolved towards a communicatecompute-control system, laying the foundations for intense use of intelligent machines and the rise of new services, interconnecting humans and machines possessing various degrees of intelligence (either natural or artificial). As recently explained in [88], future services will induce to a radical change on the conventional notions of knowing and learning, guessing and discovering. Knowledge and decisions will become a new commodity of the next generation networks and services. The idea is that, whenever communication occurs to convey meaning or to accomplish a goal, the substantial focus of communications is on the impact that the received bits have on the interpretation of the meaning intended by the transmitter or on the accomplishment of a common goal. To this end, very recent research [88] is exploring the potential of Semantic and Goal-Oriented communications to help to identify the relevant information, i.e. the information strictly necessary to recover the meaning intended by the transmitter or to accomplish a goal. Thanks to semantic communications, 6G will be able to support new types of services such as semantic services [88], seamlessly sup- porting a share of knowledge and intertwining between different kinds of intelligence. Semantic communications will remodel wireless communications from connected things to connected intelligences.

\subsection{Quantum and molecular communications}

Some very preliminary applications of quantum and molecular communications may become part of 6G infrastructure, considering the time-horizon 2030-2035 for the first deployment. The same as it has been for ML and SON in 5G, will happen to quantum and molecular communications, which will actually and extensively be employed in beyond-6G communication networks. That will be also due to the fact that these very different and novel communication paradigms will need a mature and suitable virtual network infrastructure, which will only come with 6G (as mentioned above).

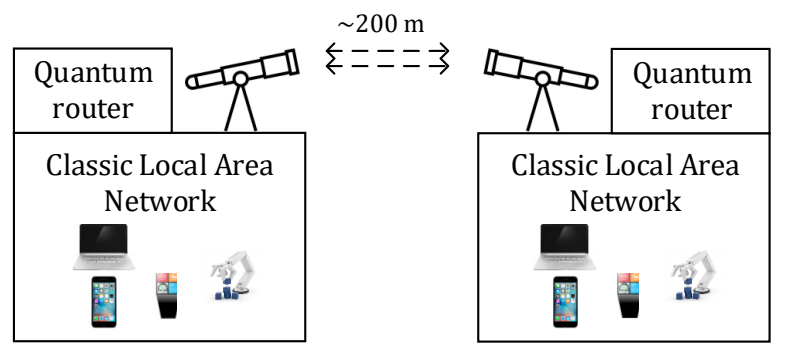

Fig. 13 - Classic local area networks interconnected by quantum routers and wireless quantum links to realise a quantum campus network.

Quantum communications [112] have been discussed for decades. While in classic communications the information is encoded into bits, in quantum communications, the information is encoded into the states of a quantum system (the so-called wavefunction). In particular, if the information is encoded into the spin of a particle, the quantum system is two-dimensional, and it is called a qubit. The qubit is an object that stores information on the unit vector of a two-dimensional complex vector space. The classic values come from the two standard basis vectors. Next, by considering systems composed of multiple correlated qubits (entangled), it is possible to see a dependency after measurement, which is independent of the distance among the various qubits. The benefits of secure communications through Quantum Key Distribution (QKD) and quantum entanglement are now clearer. In fact, some initial applications of QKD will eventually be able to fall into the $6 \mathrm{G}$ final part of design and standardization. But, beyond that, there are other ways to use quantum entanglement for communication networks. Some examples are synchronisation between communication participants (important for secure and efficient communication protocols), latency-free processing of data in the communication network during transport or simply higher data rates. Quantum tech- 
nologies bring advantages to all kinds of communications in fixed networks, cellular mobile communications, and even satellites. Quantum communications will realise communication networks quite more secure against hostile attacks from the outside. The combination of quantum computing and quantum communications, towards the so-called quantum Internet, will be an exciting field of research in this regard but it will be ready for beyond $6 \mathrm{G}$ networks. In fact, the pillar of quantum communication networks is the distribution of entangled systems among the communicating nodes. However, the distribution is strongly limited by the attenuation and the coupling, implying the reduction of quality according to the distance. That is why significant effort is devoted to the design and realisation of the so-called quantum repeaters. Nevertheless, quantum repeaters need the theoretical and practical realisation of reliable quantum memories and errorcorrecting schemes. These technologies will hardly be ready for massive deployment and integration into 6G. Because of that, the quantum communication networks that will be realisable in $6 \mathrm{G}$ will be quantum LANs and quantum campus networks. Fig. 13 depicts a conceptual schematic of a quantum campus network. Classic LANs (and also classic-quantum LANs) are equipped with quantum routers, responsible for creating and distributing entanglement among the quantum nodes. If these routers communicate wirelessly, they require telescopes to reveal the photons.

Side by side, another important emerging communication paradigm refers to nano-scale and molecular communications [113]. Instead of using electromagnetic waves, in molecular communications, the information is mainly sent via the diffusion of molecules through a specific channel. Additionally, specific molecules can be employed to carry chemo-signals or instead molecular structures containing information. In this way, molecular carriers can set up multiple independent channels using the same medium. If molecules are biological, these communications are labelled 'nano-bio'. Nano-biocommunications are the main technology to realise intrabody networks.

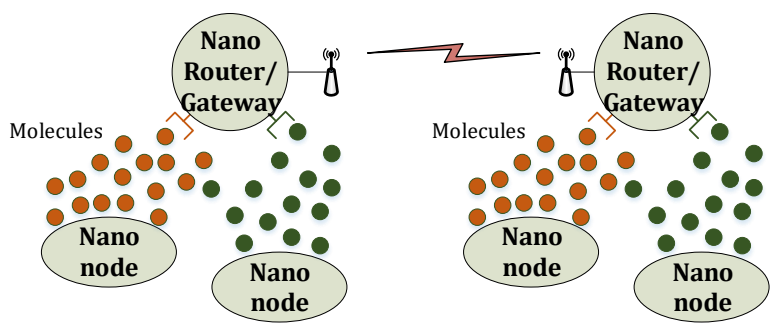

Fig. 14 - Hybrid scenario, in which molecular communications via diffusion are combined with usual wireless communication technologies.
Nano-bio-communications can fall into five categories according to the channel characteristics: Diffusion-based, molecules are immersed in a fluid and they freely diffuse; wired active, molecules move in predefined microtubules; wireless active, using bacteria carrying messages in their DNA or using nanorods (e.g. platinum or gold) exploiting the chemical energy of the environment; physical contactbased, communication happens via contacts; Förster resonance energy transfer (FRET)-based employing specific molecules called fluorophores, which can be excited by either optical or chemical stimuli. Side by side, another classification of nano-bio communications is possible according to the way molecules propagate [114]. First, walkway-based communications follow predefined paths, next flow-based communications release molecules in a fluid and guide them using currents or flows, and finally diffusion-based communications. Even if significant research is ongoing to design and develop the first molecular communication to empower eHealth and realising intra-body networks, the paradigm of the Internet of BioNano-Things [115] is still far from being reality. Fig. 14 depicts the combination of molecular and classic communication technologies, for example, in industrial scenarios and agriculture. Various nano-nodes emit molecularbased signals via diffusion to a nano-router, which can forward the information. As an example, this can be a possible use case of monitoring specific conditions in chemical industry. Next, nano-routers can also be gateways and aggregation points for then being able to convert the molecular signals into classic ones. By equipping these gateways with classic wireless interfaces, the information sent by molecules can be transmitted through longer distances, for example in industrial campus networks or via the Internet. As it has just been said for quantum communications, we believe that the technical issues, and the ethics problems involved within biomolecular communications, will make molecular communications available for networks beyond 6G.

\section{CONCLUSION}

This article started describing the main characteristics of LTE wireless cellular networks in parallel to the emerging softwarization and computing paradigms, that were causing the inherent change of wireless networks towards the advent of $5 \mathrm{G}$. Next, the critical presentation of $5 \mathrm{G}$ architectural characteristics, KPI, use cases, and standardised technologies was important to show and to motivate in detail what applications and services will lead to 6G communication networks. For this purpose, the article surveyed the research trends in the literature about $6 \mathrm{G}$, in order to understand what the 6G system requirements, KPI, innovative architectures and applications will be. The final part of this work critically discussed the main aspects of $6 \mathrm{G}$ in the authors' vision, in respect of what the literature has been proposing and what $5 \mathrm{G}$ has become in the current standardization phase. 


\section{ACKNOWLEDGEMENT}

This work has been partly funded by the European Commission through the H2020 projects Hexa-X (Grant Agreement no. 101015956), DEDICAT 6G (Grant Agreement no. 101016499) and RISE-6G. This work has also been partially funded by the German Research Foundation (DFG, Deutsche Forschungsgemeinschaft) as part of Germany's Excellence Strategy - EXC2050/1 - Project ID 390696704 - Cluster of Excellence "Centre for Tactile Internet with Human-in-the-Loop" (CeTI) of Technische Universität Dresden.

\section{ACRONYMS}

$$
\begin{array}{ll}
\text { 3GPP } & \text { 3rd Generation Partnership } \\
& \text { Project }
\end{array}
$$

$\begin{array}{ll}\text { AF } & \text { Application Function } \\ \text { AI } & \begin{array}{l}\text { Artificial Intelligence } \\ \text { Autonomic Management and } \\ \text { AMC }\end{array} \\ \text { Control } \\ \text { Access and Mobility Manage- } \\ \text { ment Function } \\ \text { API } & \begin{array}{l}\text { Application Programming } \\ \text { Interface }\end{array} \\ \text { AR } & \text { Augmented Reality } \\ \text { ARQ } & \text { Automatic Repeat reQuest } \\ \text { ASIC } & \text { Application-specific Integrated } \\ & \text { Circuit }\end{array}$

BBU Baseband Unit

CAPEX Capital Expenditure

CN Core Network

CPRI Common Public Radio Interface

C-RAN Cloud Radio Access Network

CU Centralised Unit

D2D Device-to-Device

DE Decision-making-Element

DeNB Donor eNodeB

DRB Data Radio Bearer

DSP Digital Signal Processor

DU Distributed Unit

EM Element Management

eMBB enhanced Mobile Broadband

ENI Experiential Networked Intelligence

EPC Evolved Packet Core

EPS Evolved Packet System

ETSI European Telecommunications Standards Institute

E-UTRAN Evolved Universal Terrestrial

\begin{tabular}{|c|c|}
\hline FDD & Frequency-Division Duplex \\
\hline FEC & Forward Error Correction \\
\hline FFT & Fast Fourier Transform \\
\hline FPGA & Field Programmable Gate Array \\
\hline GANA & $\begin{array}{l}\text { Generic Autonomic Network- } \\
\text { ing Architecture }\end{array}$ \\
\hline GERAN & GSM RAN \\
\hline GNSS & $\begin{array}{l}\text { Global Navigation Satellite } \\
\text { System }\end{array}$ \\
\hline GOPS & Giga-Operations Per Second \\
\hline GPRS & General Packet Radio Service \\
\hline GSM & $\begin{array}{l}\text { Global System for Mobile Com- } \\
\text { munications }\end{array}$ \\
\hline HAP & High-Altitude Platforms Hybrid \\
\hline HARQ & $\begin{array}{l}\text { Automatic Repeat } \\
\text { Request }\end{array}$ \\
\hline HEO & High Earth Orbit \\
\hline HSPA & High Speed Packet Access \\
\hline HSS & Home Subscriber Server \\
\hline IaaS & Infrastructure-as-a-Service \\
\hline IC & Infrastructure SDN Controller \\
\hline IEEE & $\begin{array}{l}\text { Institute of Electrical and Elec- } \\
\text { tronics Engineers }\end{array}$ \\
\hline IETF & $\begin{array}{l}\text { Internet Engineering Task } \\
\text { Force }\end{array}$ \\
\hline IMS & IP-Multimedia Subsystem \\
\hline IMT & $\begin{array}{l}\text { International Mobile Telecom- } \\
\text { munications }\end{array}$ \\
\hline IMT-2000 & $\begin{array}{l}\text { International Mobile } \\
\text { Telecommunications- } 2000\end{array}$ \\
\hline IoT & Internet of Things \\
\hline IP & Internet Protocol \\
\hline IRS & Intelligent Reflecting Surfaces \\
\hline ISG & Industry Specification Group \\
\hline ISO & $\begin{array}{l}\text { International Organization for } \\
\text { Standardization }\end{array}$ \\
\hline ITU & $\begin{array}{l}\text { International Telecommunica- } \\
\text { tion Union }\end{array}$ \\
\hline ITU-R & $\begin{array}{l}\text { ITU Radiocommunication } \\
\text { Sector }\end{array}$ \\
\hline ITU-T & $\begin{array}{l}\text { ITU Telecommunication Stan- } \\
\text { dardization Sector }\end{array}$ \\
\hline JSON & JavaScript Object Notation \\
\hline KPI & Key Performance Indicators \\
\hline KVI & Key Value Indicators \\
\hline LAN & Local Area Network \\
\hline LEO & Low Earth Orbit \\
\hline LTE & Long Term Evolution \\
\hline
\end{tabular}
Radio Access Network 


\begin{tabular}{|c|c|c|c|}
\hline MAC & Medium Access Control & RLC & Radio Link Control \\
\hline \multirow[t]{2}{*}{ MANO } & \multirow{2}{*}{$\begin{array}{l}\text { Management and Orchestra- } \\
\text { tion }\end{array}$} & RN & Relay Node \\
\hline & & $\mathrm{RRC}$ & Radio Resource Control \\
\hline \multirow[t]{2}{*}{ MBRLLC } & \multirow{2}{*}{$\begin{array}{l}\text { Mobile-Broadband Reliable } \\
\text { Low-latency Communication }\end{array}$} & RRH & Remote Radio Head \\
\hline & & & \\
\hline MEC & Mobile Edge Computing & & \\
\hline MEO & Medium Earth Orbit & SA & Stand-Alone \\
\hline MIMO & $\begin{array}{l}\text { Multiple-Input } \quad \text { Multiple- } \\
\text { Output }\end{array}$ & $\begin{array}{l}\text { SaaS } \\
\text { SAES }\end{array}$ & $\begin{array}{l}\text { Software-as-a-Service } \\
\text { System Architecture Evolution }\end{array}$ \\
\hline ML & Machine Learning & & Specification \\
\hline MME & Mobility Management Entity & SBA & Service-Based Architecture \\
\hline mMTC & $\begin{array}{l}\text { massive Machine-Type Com- } \\
\text { munication }\end{array}$ & SDL & $\begin{array}{l}\text { Supplementary Downlink } \\
\text { Software-Defined Networking }\end{array}$ \\
\hline MOS & Mean Opinion Score & SDPA & $\begin{array}{l}\text { Service Data Adaptation Proto- } \\
\text { col }\end{array}$ \\
\hline NAS & Non-Access Stratum & SDR & Software-Defined Radio \\
\hline NFV & $\begin{array}{l}\text { Network Function Virtualisa- } \\
\text { tion }\end{array}$ & $\begin{array}{l}\text { SFC } \\
\text { S-GW }\end{array}$ & $\begin{array}{l}\text { Service Function Chaining } \\
\text { Serving Gateway }\end{array}$ \\
\hline NFVI & $\begin{array}{l}\text { Network Function Virtualiza- } \\
\text { tion Infrastructure }\end{array}$ & $\begin{array}{l}\text { SIP } \\
\text { SLA }\end{array}$ & $\begin{array}{l}\text { Session Initiation Protocol } \\
\text { Service Level Agreement }\end{array}$ \\
\hline NMGM & $\begin{array}{l}\text { New Generation Mobile Net- } \\
\text { works }\end{array}$ & $\begin{array}{l}\text { SMF } \\
\text { SON }\end{array}$ & $\begin{array}{l}\text { Session Management Function } \\
\text { Self-Organising Networks }\end{array}$ \\
\hline NMS & Network Management System & SUL & Supplementary Uplink \\
\hline $\begin{array}{l}\text { NR } \\
\text { NSA }\end{array}$ & $\begin{array}{l}\text { New Radio } \\
\text { Non-Stand-Alone }\end{array}$ & TaHiL & $\begin{array}{l}\text { Tactile Internet with Human- } \\
\text { in-the-Loop }\end{array}$ \\
\hline OFDMA & $\begin{array}{l}\text { Orthogonal Frequency- } \\
\text { Division Multiple Access }\end{array}$ & $\begin{array}{l}\text { TC } \\
\text { TCP } \\
\text { TDD }\end{array}$ & $\begin{array}{l}\text { Tenant SDN Controller } \\
\text { Transmission Control Protocol } \\
\text { Time-Division Duplex }\end{array}$ \\
\hline ONF & Open Network Foundation & TDMA & $\begin{array}{l}\text { 11me-DIVISIon Duplex } \\
\text { Time-Division Multiple Access }\end{array}$ \\
\hline$\mu O N O S$ & $\begin{array}{l}\text { Micro Open Network Operating } \\
\text { System }\end{array}$ & TLS & Transport Layer Security \\
\hline OPEX & Operational Expenditure & UAV & Unmanned Aerial Vehicles \\
\hline OSI & Open Systems Interconnection & $\begin{array}{l}\text { UDP } \\
\text { UE }\end{array}$ & $\begin{array}{l}\text { User Datagram Protocol } \\
\text { User Equipment }\end{array}$ \\
\hline PaaS & Platform-as-a-Service & uHDD & ultra-High Data Density \\
\hline PDCP & $\begin{array}{l}\text { Packet Data Convergence } \\
\text { Protocol }\end{array}$ & uHSLLC & $\begin{array}{l}\text { ultra-High-Speed Low-latency } \\
\text { Communications }\end{array}$ \\
\hline PDN-GW & Packet Data Network Gateway & uMTC & ultra-reliable Machine-Type \\
\hline PDU & Protocol Data Unit & & Communications \\
\hline PPS & Programmable Protocol Stack & UMTS & Universal Mobile Telecommu- \\
\hline PRB & Physical Resource Block & & nications System \\
\hline PSTN & $\begin{array}{l}\text { Public Switched Telephone } \\
\text { Network }\end{array}$ & uMUB & $\begin{array}{l}\text { ubiquitous Mobile Ultra- } \\
\text { Broadband }\end{array}$ \\
\hline & & UPF & User Plane Function \\
\hline QKD & Quantum Key Distribution & URLLC & Ultra-Reliable Low-Latency \\
\hline QoE & Quality-of-Experience & & Communication \\
\hline QoP & Quality-of-Perception & UTRAN & UMTS Terrestrial Radio Access \\
\hline QoPE & Quality-of-Physical-Experience & & Network \\
\hline QoS & Quality-of-Service & & \\
\hline RAN & Radio Access Network & VIM & $\begin{array}{l}\text { Virtualized Infrastructure Man- } \\
\text { ager }\end{array}$ \\
\hline REST & Representational state transfer & VLC & Visible Light Communications \\
\hline RIS & $\begin{array}{l}\text { Reconfigurable Intelligent } \\
\text { Surface }\end{array}$ & VNFM & $\begin{array}{l}\text { Virtual Network Function Man- } \\
\text { ager }\end{array}$ \\
\hline
\end{tabular}


VR Virtual Reality

WCDMA Wideband Code-Division Multiple Access

WLAN Wireless Local Area Network

\section{REFERENCES}

[1] F. H. P. Fitzek, F. Granelli, and P. Seeling, Eds., Computing in Communication Networks - From Theory to Practice, 1st ed., ser. 1. Elsevier, Jan. 1, 2020, vol. 1, ISBN: 978-0128204887.

[2] Hexa-X. (Feb. 2021). "D1.1 - 6G Vision, use cases and key societal values," [Online]. Available: https : / / hexa-x.eu/wp-content/uploads / 2021/02/Hexa-X_D1.1.pdf (visited on 2021).

[3] RISE-6G. 0. “RISE-6G: Reconfigurable Intelligent Sustainable Environments for 6G Wireless Networks.," [Online]. Available: https : / / 5g-ppp . $\mathrm{eu} / \mathrm{rise}-6 \mathrm{~g} /$ (visited on 2021).

[4] DEDICAT 6G. 0. "DEDICAT 6G: Dynamic coverage Extension and Distributed Intelligence for human Centric Applications with assured security, privacy, and Trust: from 5G to 6G.," [Online]. Available: https : / /5g-ppp . eu/dedicat-6g/ (visited on 2021).

[5] Next G Alliance. (). "Next G Alliance: Building the Foundation for North American Leadership in 6G and Beyond," [Online]. Available: https : // nextgalliance.org/ (visited on 2021).

[6] M. Olsson, S. Sultana, S. Rommer, L. Frid, and C. Mulligan, SAE and the Evolved Packet Core: Driving the Mobile Broadband Revolution. Academic Press, 2009, ISBN: 9780123748263.

[7] R. Bassoli, H. Marques, J. Rodriguez, C. Gruet, and R. Tafazolli, "Enhanced authentication for WLANEPS interworking systems," Electronics Letters, vol. 51, no. 19, pp. 1544-1546, 2015. DoI: https : / / doi .org/10 . 1049/el . 2014 . 4542. eprint: https : / / ietresearch . onlinelibrary . wiley.com/doi/pdf/10.1049/el . 2014.4542. [Online]. Available: https : / / ietresearch . onlinelibrary . wiley . com / doi / abs / 10 . 1049/el.2014.4542.

[8] A. Asadi, Q. Wang, and V. Mancuso, "A Survey on Device-to-Device Communication in Cellular Networks," IEEE Communications Surveys \& Tutorials, vol. 16, no. 4, pp.1801-1819, 2014. DoI: 10.1109/ COMST . 2014.2319555.

[9] J. Liu, N. Kato, J. Ma, and N. Kadowaki, "Deviceto-Device Communication in LTE-Advanced Networks: A Survey," IEEE Communications Surveys \& Tutorials, vol. 17, no. 4, pp. 1923-1940, 2015. DoI: 10.1109/COMST . 2014. 2375934.
[10] A. Jarwan, A. Sabbah, M. Ibnkahla, and O. Issa, "Lte-based public safety networks: A survey," IEEE Communications Surveys Tutorials, vol. 21, no. 2, pp. 1165-1187, 2019. DoI: 10 . $1109 /$ COMST . 2019.2895658.

[11] B. Van Der Bergh, A. Chiumento, and S. Pollin, "LTE in the sky: trading off propagation benefits with interference costs for aerial nodes," IEEE Communications Magazine, vol. 54, no. 5, pp. 44-50, 2016. DOI: 10.1109/MCOM. 2016.7470934.

[12] N. Carr, Cloud computing, ser. Encyclopædia Britannica. Encyclopædia Britannica, inc., Feb. 2021. [Online]. Available: https : //www . britannica . com/technology/cloud-computing.

[13] M. Chiosi, D. Clarke, P. Willis, A. Reid, J. Feger, M. Bugenhagen, W. Khan, M. Fargano, C. Cui, H. Deng, J. Benitez, U. Michel, H. Damker, K. Ogaki, T. Matsuzaki, M. Fukui, K. Shimano, D. Delisle, Q. Loudier, C. Kolias, I. Guardini, E. Demaria, R. Minerva, A. Manzalini, D. L. adn Francisco Javier Ramón Salguero, F. Ruhl, and P. Sen. (Oct. 2012). "Network Functions Virtualisation - An Introduction, Benefits, Enablers, Challenges \& Call for Action," [Online]. Available: https : / / docbox . etsi . org / isg / nfv / open / Publications pdf/White $\% 5 \mathrm{C} \% 20$ Papers/NFV_White_Paper 1 _ 2012.pdf (visited on 2021).

[14] ITU-T FG IMT-2020. (Dec. 2016). "Draft Terms and definitions for IMT-2020," [Online]. Available: https : / / www . itu . int / en / ITU - T / focusgroups/imt-2020/Pages/default . aspx (visited on 2021).

[15] A. Laghrissi and T. Taleb, "A Survey on the Placement of Virtual Resources and Virtual Network Functions," IEEE Communications Surveys \& Tutorials, vol. 21, no. 2, pp. 1409-1434, 2019. DoI: 10 . 1109/COMST . 2018. 2884835.

[16] ITU-R. (Sep. 2015). "ITU-R M.2083-0 - IMT Vision - Framework and overall objectives of the future development of IMT for 2020 and beyond," [Online]. Available: https : / / www . itu . int/dms _ pubrec/itu-r/rec/m/R-REC-M . 2083-0201509-I! !PDF-E.pdf (visited on 2021).

[17] NGMN Alliance. (Feb. 2015). "NGMN 5G Initiative White Paper," [Online]. Available: https : / / www . ngmn . org / wp - content / uploads / NGMN_5G_ White_Paper_V1_0.pdf (visited on 2021).

[18] J. G. Andrews, S. Buzzi, W. Choi, S. V. Hanly, A. Lozano, A. C. K. Soong, and J. C. Zhang, "What Will $5 \mathrm{G}$ Be?" IEEE Journal on Selected Areas in Communications, vol. 32, no. 6, pp. 1065-1082, 2014. DoI: 10.1109/JSAC . 2014. 2328098. 
[19] M. Agiwal, A. Roy, and N. Saxena, "Next Generation 5G Wireless Networks: A Comprehensive Survey," IEEE Communications Surveys \& Tutorials, vol. 18, no. 3, pp. 1617-1655, 2016. DoI: 10 . 1109/COMST . 2016. 2532458.

[20] GSMA. (Dec. 2014). "Understanding 5G: Perspectives on future technological advancements in mobile," [Online]. Available: https : / / www . gsma . com / futurenetworks / wp content / uploads / 2015 / 01 / 2014 - 12 08-c88a32b3c59a11944a9c4e544fee7770.pdf (visited on 2021).

[21] E. Calvanese Strinati, S. Barbarossa, J. L. GonzalezJimenez, D. Ktenas, N. Cassiau, L. Maret, and C. Dehos, “6G: The Next Frontier: From Holographic Messaging to Artificial Intelligence Using Subterahertz and Visible Light Communication," IEEE Vehicular Technology Magazine, vol. 14, no. 3, pp. 42-50, 2019. DOI: 10 . 1109 / MVT . 2019 . 2921162.

[22] S. Chen, Y. Liang, S. Sun, S. Kang, W. Cheng, and M. Peng, "Vision, requirements, and technology trend of 6g: How to tackle the challenges of system coverage, capacity, user datarate and movement speed," IEEE Wireless Communications, vol. 27 , no. 2 , pp. 218-228, 2020. DoI: 10.1109/MWC.001.1900333.

[23] ETSI. (Jan. 2010). "ETSI TR 102643 - Human Factors (HF); Quality of Experience (QoE) requirements for real-time communication services," [Online]. Available: https : / / www . etsi . org / deliver/etsi_tr/102600_102699/102643/ $01.00 .02 \_60 /$ tr_102643v010002p.pdf (visited on 2021).

[24] J. Navarro-Ortiz, P. Romero-Diaz, S. Sendra, P. Ameigeiras, J. J. Ramos-Munoz, and J. M. LopezSoler, "A Survey on 5G Usage Scenarios and Traffic Models," IEEE Communications Surveys \& Tutorials, vol. 22, no. 2, pp. 905-929, 2020. DoI: 10 . 1109/COMST . 2020. 2971781.

[25] J. Halpern and C. Pignataro, Eds. (Oct. 2015). "Service Function Chaining (SFC) Architecture," [Online]. Available: https : / / tools . ietf . org / html/rf c7665 (visited on 2021).

[26] X. Foukas, G. Patounas, A. Elmokashfi, and M. K. Marina, "Network Slicing in 5G: Survey and Challenges," IEEE Communications Magazine, vol. 55, no. 5, pp. 94-100, 2017. DOI: 10 . 1109 / MCOM . 2017.1600951.

[27] I. Afolabi, T. Taleb, K. Samdanis, A. Ksentini, and H. Flinck, "Network Slicing and Softwarization: A Survey on Principles, Enabling Technologies, and Solutions," IEEE Communications Surveys \& Tutorials, vol. 20, no. 3, pp. 2429-2453, 2018. DoI: 10 . 1109/COMST . 2018. 2815638.
[28] R. Su, D. Zhang, R. Venkatesan, Z. Gong, C. Li, F. Ding, F. Jiang, and Z. Zhu, "Resource Allocation for Network Slicing in 5G Telecommunication Networks: A Survey of Principles and Models," IEEE Network, vol. 33, no. 6, pp. 172-179, 2019. DoI: 10.1109/MNET . 2019.1900024.

[29] S. Zhang, "An Overview of Network Slicing for 5G," IEEE Wireless Communications, vol. 26, no. 3, pp. 111-117, 2019. DoI: 10 . 1109 / MWC . 2019 . 1800234.

[30] U. Dötsch, M. Doll, H. Mayer, F. Schaich, J. Segel, and P. Sehier, "Quantitative analysis of split base station processing and determination of advantageous architectures for LTE," Bell Labs Technical Journal, vol. 18, no. 1, pp. 105-128, 2013. DoI: 10 . 1002/bltj. 21595.

[31] M. Jaber, M. A. Imran, R. Tafazolli, and A. Tukmanov, "5G Backhaul Challenges and Emerging Research Directions: A Survey," IEEE Access, vol. 4, pp. 1743-1766, 2016. DOI: 10 . 1109 / ACCESS . 2016.2556011.

[32] F. Bonomi, R. Milito, J. Zhu, and S. Addepalli, "Fog Computing and Its Role in the Internet of Things," in Proceedings of the First Edition of the MCC Workshop on Mobile Cloud Computing, ser. MCC '12, Helsinki, Finland: Association for Computing Machinery, 2012, pp. 13-16, ISBN: 9781450315197. DoI: $10.1145 / 2342509.2342513$. [Online]. Available: https : / / doi .org / 10 . 1145/2342509 . 2342513.

[33] Y. Mao, C. You, J. Zhang, K. Huang, and K. B. Letaief, "A Survey on Mobile Edge Computing: The Communication Perspective," IEEE Communications Surveys \& Tutorials, vol. 19, no. 4, pp. 2322-2358, 2017. DOI: 10.1109/COMST . 2017 . 2745201.

[34] J. Ren, D. Zhang, S. He, Y. Zhang, and T. Li, "A survey on end-edge-cloud orchestrated network computing paradigms: Transparent computing, mobile edge computing, fog computing, and cloudlet," ACM Comput. Surv., vol. 52, no. 6, Oct. 2019, ISSN: 0360-0300. DOI: 10 . $1145 /$ 3362031. [Online]. Available: https ://doi .org/ $10.1145 / 3362031$.

[35] C. Puliafito, E. Mingozzi, F. Longo, A. Puliafito, and O. Rana, "Fog computing for the internet of things: A survey," ACM Transactions on Internet Technology, vol. 19, no. 2, Apr. 2019, ISSN: 15335399. DoI: 10.1145/3301443. [Online]. Available: https://doi.org/10.1145/3301443.

[36] ETSI. (Jan. 2019). "Multi-access Edge Computing (MEC); Terminology," [Online]. Available: https : //www . etsi.org/deliver/etsi_gs/MEC/001_ 099/001/02.01.01_60/gs_MEC001v020101p. pdf (visited on 2021). 
[37] ETSI. (Jul. 2020). "Harmonizing standards for edge computing - A synergized architecture leveraging ETSI ISG MEC and 3GPP specifications," [Online]. Available: https : / / www . etsi . org / images / files / ETSIWhitePapers / ETSI_ wp36 _ Harmonizing - standards - for - edge computing.pdf (visited on 2021).

[38] 3GPP. (Sep. 2019). "3rd Generation Partnership Project; Technical Specification Group Services and System Aspects; Release 15 Description; Summary of Rel-15 Work Items (Release 15)," [Online]. Available: https : / / portal . 3gpp . org / desktopmodules / Specifications / SpecificationDetails . aspx?specificationId=3389 (visited on 2021).

[39] ITU. (Feb. 2021). "Beyond 5G: What's next for IMT?" [Online]. Available: https : / / www . itu . int / en / myitu / News / 2021 / 02 / 02 / 09 / 20 / Beyond - 5G - IMT - 2020 - update - new Recommendation (visited on 2021).

[40] M. Giordani, M. Polese, M. Mezzavilla, S. Rangan, and M. Zorzi, "Toward 6G Networks: Use Cases and Technologies," IEEE Communications Magazine, vol. 58, no. 3, pp. 55-61, 2020. DoI: 10.1109/ MCOM.001.1900411.

[41] R. Minerva, G. M. Lee, and N. Crespi, "Digital Twin in the IoT Context: A Survey on Technical Features, Scenarios, and Architectural Models," Proceedings of the IEEE, vol. 108, no. 10, pp. 17851824, 2020. DOI: 10 . 1109 / JPROC . 2020. 2998530.

[42] D. Ross, "Digital twinning [information technology virtual reality]," Engineering Technology, vol. 11, no. 4, pp. 44-45, 2016. Dor: 10.1049/et. 2016.0403.

[43] A. El Saddik, "Digital twins: The convergence of multimedia technologies," IEEE MultiMedia, vol. 25, no. 2, pp. 87-92, 2018. DoI: 10 . $1109 /$ MMUL . 2018.023121167.

[44] R. Saracco, "Digital twins: Bridging physical space and cyberspace," Computer, vol. 52, no. 12, pp. 5864, 2019. DOI: 10.1109/MC . 2019. 2942803.

[45] A. Rasheed, O. San, and T. Kvamsdal, "Digital twin: Values, challenges and enablers from a modeling perspective," IEEE Access, vol. 8, pp. 21980 22 012, 2020. DOI: 10 . 1109 / ACCESS . 2020 . 2970143.

[46] F. Tao, H. Zhang, A. Liu, and A. Y. C. Nee, "Digital twin in industry: State-of-the-art," IEEE Transactions on Industrial Informatics, vol. 15, no. 4, pp. 2405-2415, 2019. DOI: 10 . 1109/TII . 2018. 2873186.
[47] H. Laaki, Y. Miche, and K. Tammi, "Prototyping a digital twin for real time remote control over mobile networks: Application of remote surgery," IEEE Access, vol. 7, pp. $20325-20336,2019$. DoI: 10.1109/ACCESS. 2019.2897018.

[48] S. H. Khajavi, N. H. Motlagh, A. Jaribion, L. C. Werner, and J. Holmström, "Digital twin: Vision, benefits, boundaries, and creation for buildings," IEEE Access, vol. 7, pp. 147 406-147 419, 2019. DOI: 10.1109/ACCESS . 2019.2946515.

[49] B. R. Barricelli, E. Casiraghi, and D. Fogli, "A survey on digital twin: Definitions, characteristics, applications, and design implications," IEEE Access, vol. 7, pp. 167 653-167 671, 2019. DoI: 10 . 1109/ACCESS . 2019. 2953499.

[50] G. Bachelor, E. Brusa, D. Ferretto, and A. Mitschke, "Model-based design of complex aeronautical systems through digital twin and thread concepts," IEEE Systems Journal, vol. 14, no. 2, pp. 1568-1579, 2020. DOI: 10 . 1109 / JSYST . 2019.2925627.

[51] T. R. Wanasinghe, L. Wroblewski, B. K. Petersen, R. G. Gosine, L. A. James, O. De Silva, G. K. I. Mann, and P. J. Warrian, "Digital twin for the oil and gas industry: Overview, research trends, opportunities, and challenges," IEEE Access, vol. 8, pp. $104175-104197,2020$. DOI: 10 . 1109 / ACCESS . 2020 . 2998723.

[52] F. Laamarti, H. F. Badawi, Y. Ding, F. Arafsha, B. Hafidh, and A. E. Saddik, "An ISO/IEEE 11073 Standardized Digital Twin Framework for Health and Well-Being in Smart Cities," IEEE Access, vol. 8, pp. 105 950-105 961, 2020. DoI: 10.1109/ ACCESS . 2020.2999871.

[53] B. Zong, C. Fan, X. Wang, X. Duan, B. Wang, and J. Wang, "6G Technologies: Key Drivers, Core Requirements, System Architectures, and Enabling Technologies," IEEE Vehicular Technology Magazine, vol. 14, no. 3, pp. 18-27, 2019. DoI: 10.1109/ MVT . 2019. 2921398.

[54] W. Saad, M. Bennis, and M. Chen, "A Vision of 6G Wireless Systems: Applications, Trends, Technologies, and Open Research Problems," IEEE Network, vol. 34, no. 3, pp. 134-142, 2020. DoI: 10 . 1109/MNET . 001.1900287.

[55] K. B. Letaief, W. Chen, Y. Shi, J. Zhang, and Y. A. Zhang, "The Roadmap to 6G: AI Empowered Wireless Networks," IEEE Communications Magazine, vol. 57, no. 8, pp. 84-90, 2019. DoI: 10 . 1109 / MCOM . 2019.1900271.

[56] Z. Zhang, Y. Xiao, Z. Ma, M. Xiao, Z. Ding, X. Lei, G. K. Karagiannidis, and P. Fan, "6G Wireless Networks: Vision, Requirements, Architecture, and Key Technologies," IEEE Vehicular Technology Magazine, vol. 14, no. 3, pp. 28-41, 2019. DOI: $10.1109 /$ MVT . 2019.2921208. 
[57] V. Ziegler and S. Yrjola, "6G Indicators of Value and Performance," in 2020 2nd 6G Wireless Summit (6G SUMMIT), 2020, pp. 1-5. DOI: 10.1109 / 6GSUMMIT49458.2020.9083885.

[58] Q. Bi, "Ten Trends in the Cellular Industry and an Outlook on 6G," IEEE Communications Magazine, vol. 57, no. 12 , pp. 31-36, 2019. DoI: 10.1109 / MCOM.001.1900315.

[59] V. S. Pendyala, S. S. Y. Shim, and C. Bussler, "The web that extends beyond the world," Computer, vol. 48, no. 5, pp. 18-25, 2015. DoI: 10.1109/MC . 2015.150.

[60] E. Calvanese Strinati, S. Barbarossa, T. Choi, A. Pietrabissa, A. Giuseppi, E. De Santis, J. Vidal, Z. Becvar, T. Haustein, C. Nicolas, F. Costanzo, J. Kim, and I. Kim, "6G in the sky: On-demand intelligence at the edge of 3D networks," ETRI Journal, vol. 10.4218/etrij.2020-0205, 2020.

[61] M. Giordani and M. Zorzi, "Non-Terrestrial Networks in the 6G Era: Challenges and Opportunities," IEEE Network, pp. 12-19, 2020. DoI: 10 . 1109/MNET.011.2000493.

[62] X. Huang, J. A. Zhang, R. P. Liu, Y. J. Guo, and L. Hanzo, "Airplane-Aided Integrated Networking for 6G Wireless: Will It Work?" IEEE Vehicular Technology Magazine, vol. 14, no. 3, pp. 84-91, 2019. DOI: 10.1109/MVT . 2019.2921244.

[63] E. Yaacoub and M. Alouini, "A Key 6G Challenge and Opportunity-Connecting the Base of the Pyramid: A Survey on Rural Connectivity," Proceedings of the IEEE, vol. 108, no. 4, pp. 533-582, 2020. DOI: 10.1109/JPROC. 2020. 2976703.

[64] J. Rodriguez, G. P. Koudouridis, X. Gelabert, M. Tayyab, R. Bassoli, F. H. P. Fitzek, R. Torre, R. Abd-Alhameed, M. Sahedin, I. Elfergani, S. Irum, G. Schulte, P. Diogo, F. Marzouk, M. de Ree, G. Mantas, and I. Politis, "Secure virtual mobile small cells: A stepping stone towards 6g," IEEE Communications Standards Magazine, pp. 1-15, 2021. DOI: 10.1109/MCOMSTD .001.2000019.

[65] S. Zhang, J. Liu, H. Guo, M. Qi, and N. Kato, "Envisioning Device-to-Device Communications in 6G," IEEE Network, vol. 34, no. 3, pp. 86-91, 2020. DoI: 10.1109/MNET.001.1900652.

[66] H. Yang, A. Alphones, Z. Xiong, D. Niyato, J. Zhao, and $\mathrm{K} . \mathrm{Wu}$, "Artificial-Intelligence-Enabled Intelligent 6G Networks," IEEE Network, vol. 34, no. 6, pp. 272-280, 2020. DOI: 10 . 1109 / MNET . 011 . 2000195.
[67] J. Du, C. Jiang, J. Wang, Y. Ren, and M. Debbah, "Machine Learning for 6G Wireless Networks: Carrying Forward Enhanced Bandwidth, Massive Access, and Ultrareliable/Low-Latency Service," IEEE Vehicular Technology Magazine, vol. 15, no. 4, pp. 122-134, 2020. DoI: 10 . 1109 / MVT . 2020.3019650 .

[68] C. She, R. Dong, Z. Gu, Z. Hou, Y. Li, W. Hardjawana, C. Yang, L. Song, and B. Vucetic, "Deep Learning for Ultra-Reliable and Low-Latency Communications in 6G Networks," IEEE Network, vol. 34, no. 5, pp. 219-225, 2020. DOI: 10 . 1109 / MNET . 011 . 1900630.

[69] R. Shafin, L. Liu, V. Chandrasekhar, H. Chen, J. Reed, and J. C. Zhang, "Artificial IntelligenceEnabled Cellular Networks: A Critical Path to Beyond-5G and 6G," IEEE Wireless Communications, vol. 27, no. 2, pp. 212-217, 2020. DoI: 10 . 1109/MWC.001.1900323.

[70] S. Han, T. Xie, C. .-L. I, L. Chai, Z. Liu, Y. Yuan, and C. Cui, "Artificial-Intelligence-Enabled Air Interface for 6G: Solutions, Challenges, and Standardization Impacts," IEEE Communications Magazine, vol. 58, no. 10, pp. 73-79, 2020. DoI: 10.1109/MCOM. 001. 2000218.

[71] Y. Xiao, G. Shi, Y. Li, W. Saad, and H. V. Poor, "Toward Self-Learning Edge Intelligence in 6G," IEEE Communications Magazine, vol. 58, no. 12, pp. 3440, 2020. DOI: 10.1109/MCOM. 001.2000388.

[72] M. Polese, J. M. Jornet, T. Melodia, and M. Zorzi, "Toward End-to-End, Full-Stack 6G Terahertz Networks," IEEE Communications Magazine, vol. 58, no. 11, pp. 48-54, 2020. DoI: 10 . 1109 / MCOM. 001.2000224.

[73] P. Yang, Y. Xiao, M. Xiao, and S. Li, "6G Wireless Communications: Vision and Potential Techniques," IEEE Network, vol. 33, no. 4, pp. 70-75, 2019. DOI: 10.1109/MNET . 2019.1800418.

[74] K. Rikkinen, P. Kyosti, M. E. Leinonen, M. Berg, and A. Parssinen, "THz Radio Communication: Link Budget Analysis toward 6G," IEEE Communications Magazine, vol. 58, no. 11, pp. 22-27, 2020. DOI: 10.1109/MCOM.001.2000310.

[75] H. Jiang, M. Mukherjee, J. Zhou, and J. Lloret, "Channel Modeling and Characteristics for 6G Wireless Communications," IEEE Network, vol. 35, no. 1, pp. 296-303, 2021. DOI: 10 . 1109 / MNET . 011.2000348.

[76] M. Matthaiou, O. Yurduseven, H. Q. Ngo, D. Morales-Jimenez, S. L. Cotton, and V. F. Fusco, "The Road to 6G: Ten Physical Layer Challenges for Communications Engineers," IEEE Communications Magazine, vol. 59, no. 1, pp. 64-69, 2021. DOI: $10.1109 /$ MCOM.001.2000208. 
[77] N. Chi, Y. Zhou, Y. Wei, and F. Hu, "Visible Light Communication in 6G: Advances, Challenges, and Prospects," IEEE Vehicular Technology Magazine, vol. 15, no. 4, pp. 93-102, 2020. DoI: $10.1109 /$ MVT. 2020.3017153.

[78] C. E. Shannon, "A mathematical theory of communication," The Bell system technical journal, vol. 27, no. 3, pp. 379-423, 1948.

[79] E. Calvanese Strinati and S. Barbarossa, "6G in the sky: On-demand intelligence at the edge of 3D networks," Computer Networks Journal, 2022.

[80] E. Basar, M. Di Renzo, J. De Rosny, M. Debbah, M.-S. Alouini, and R. Zhang, "Wireless communications through reconfigurable intelligent surfaces," IEEE Access, vol. 7, pp. 116 753-116 773, 2019.

[81] S. V. Hum and J. Perruisseau-Carrier, Reconfigurable reflectarrays and array lenses for dynamic antenna beam control: A review, 2013. arXiv: 1308.4593 [physics.optics].

[82] L. Di Palma, A. Clemente, L. Dussopt, R. Sauleau, P. Potier, and P. Pouliguen, "Circularly-polarized reconfigurable transmitarray in ka-band with beam scanning and polarization switching capabilities," IEEE Transactions on Antennas and Propagation, vol. 65, no. 2, pp. 529-540, 2016.

[83] J. R. Reis, M. Vala, and R. F. Caldeirinha, "Review paper on transmitarray antennas," IEEE Access, vol. 7, pp. 94 171-94 188, 2019.

[84] B. Atorf, H. Mühlenbernd, T. Zentgraf, and H. Kitzerow, "All-optical switching of a dye-doped liquid crystal plasmonic metasurface," Optics EXpress, vol. 28, no. 6, pp. 8898-8908, 2020.

[85] M. R. Hashemi, S. Cakmakyapan, and M. Jarrahi, "Reconfigurable metamaterials for terahertz wave manipulation," Reports on Progress in Physics, vol. 80, no. 9, p. 094 501, 2017.

[86] K. David and H. Berndt, "6G Vision and Requirements: Is There Any Need for Beyond 5G?" IEEE Vehicular Technology Magazine, vol. 13, no. 3, pp. 72-80, 2018. DOI: 10 . 1109 / MVT . 2018 . 2848498.

[87] F. H. P. Fitzek and P. Seeling, Why We Should NOT Talk about 6G, 2020. arXiv: 2003.02079 [cs.NI].

[88] E. Calvanese Strinati and S. Barbarossa, “6G networks: Beyond Shannon towards semantic and goal-oriented communications," Computer Netwrks Journal, vol. 190, no. 107930, 2021. DoI: DOI : $10.1016 /$ j . comnet . 2021.107930.

[89] J. Oueis and E. C. Strinati, "Uplink Traffic in Future Mobile Networks: Pulling the Alarm," in Cognitive Radio Oriented Wireless Networks, D. Noguet, K. Moessner, and J. Palicot, Eds., Springer International Publishing, 2016, pp. 583-593, ISBN: 9783-319-40352-6.
[90] T. Werthmann, H. Grob-Lipski, S. Scholz, and B. Haberland, "Task assignment strategies for pools of baseband computation units in $4 \mathrm{G}$ cellular networks," in 2015 IEEE International Conference on Communication Workshop (ICCW), Jun. 2015 pp. 2714-2720. DOI: 10 . 1109 / ICCW . 2015 . 7247589.

[91] M. Bennis, M. Debbah, and H. V. Poor, "Ultrareliable and Low-Latency Wireless Communication: Tail, Risk, and Scale," Proceedings of the IEEE, vol. 106, no. 10, pp. 1834-1853, 2018.

[92] X. Jiang, H. Shokri-Ghadikolaei, G. Fodor, E. Modiano, Z. Pang, M. Zorzi, and C. Fischione, "LowLatency Networking: Where Latency Lurks and How to Tame It," Proceedings of the IEEE, vol. 107, no. 2, pp. 280-306, 2019.

[93] F. Granelli and R. Bassoli, "Autonomic mobile virtual network operators for future generation networks," IEEE Network, vol. 32, no. 5, pp. 76-84, 2018. DOI: 10.1109/MNET . 2018.1700455.

[94] K. Chen, T. Zhang, R. D. Gitlin, and G. Fettweis, "Ultra-low latency mobile networking," IEEE Network, vol. 33, no. 2, pp. 181-187, 2019. DoI: 10 . 1109/MNET . 2018. 1800011

[95] P. Seeling and F. H. P. Fitzek, "Anticipatory Networking: Negative Latency for Ubiquitous Computing," in 2021 IEEE 18th Annual Consumer Communications Networking Conference (CCNC), 2021, pp. 1-4. DOI: 10.1109/CCNC49032. 2021. 9369624

[96] N. Bui, M. Cesana, S. A. Hosseini, Q. Liao, I. Malanchini, and J. Widmer, "A Survey of Anticipatory Mobile Networking: Context-Based Classification, Prediction Methodologies, and Optimization Techniques," IEEE Communications Surveys \& Tutorials, vol. 19, no. 3, pp. 1790-1821, 2017. DoI: 10.1109/COMST . 2017.2694140.

[97] Z. Xiang, F. Gabriel, E. U. Pérez, G. T. Nguyen, M. Reisslein, and F. H. P. Fitzek, "Reducing latency in virtual machines: Enabling Tactile Internet for human-machine co-working," IEEE Journal on Selected Areas in Communications, vol. 37, no. 5, pp. 1098-1116, May 2019.

[98] C. X. Wang, F. Haider, X. Gao, X. H. You, Y. Yang, D. Yuan, H. M. Aggoune, H. Haas, S. Fletcher, and E. Hepsaydir, "Cellular architecture and key technologies for $5 \mathrm{G}$ wireless communication networks," IEEE Communications Magazine, vol. 52, no. 2 , pp. $122-130,2014$, ISSN: 01636804. DOI: 10.1109/MCOM. 2014.6736752.

[99] R. Raheem, A. Lasebae, M. Aiash, and J. Loo, "Performance Evaluation of Mobile Users Served by Fixed and Mobile Femtocells in LTE Networks," Journal of Networking Technology, vol. 7, pp. 16-33, Mar. 2016. 
[100] A. Radwan, K. M. S. Huq, S. Mumtaz, K. F. Tsang, and J. Rodriguez, "Low-Cost On-Demand C-RAN Based Mobile Small-Cells," IEEE Access, vol. 4, pp. 2331-2339, 2016, ISSN: 21693536. DOI: 10 . 1109/ACCESS. 2016. 2563518.

[101] A. Radwan and J. Rodriguez, "Cloud of Mobile Small-cells for Higher Data-rates and Better Energy-efficiency," in European Wireless 2017; 23th European Wireless Conference, 2017, ISBN: 9783800744268.

[102] F. Tariq, M. R. A. Khandaker, K. .-K. Wong, M. A. Imran, M. Bennis, and M. Debbah, "A Speculative Study on 6G," IEEE Wireless Communications, vol. 27, no. 4, pp. 118-125, 2020. DoI: $10.1109 /$ MWC. 001.1900488.

[103] J. Meadowcroft, Sustainability, ser. Encyclopædia Britannica. Encyclopædia Britannica, inc., Apr. 2020. [Online]. Available: https : / / www . britannica.com/science/sustainability.

[104] K. .-C. Chen, S. .-C. Lin, J. .-H. Hsiao, C. .-H. Liu, A. F. Molisch, and G. P. Fettweis, "Wireless Networked Multirobot Systems in Smart Factories," Proceedings of the IEEE, pp. 1-27, 2020. DOI: 10 . 1109 / JPROC . 2020.3033753.

[105] F. H. P. Fitzek, S.-C. Li, S. Speidel, T. Strufe, M. Simsek, and M. Reisslein, Eds., Tactile Internet with Human-in-the-Loop. Academic Press, Jan. 1, 2021, published.

[106] R. Bassoli, F. Granelli, C. Sacchi, S. Bonafini, and F. H. P. Fitzek, "CubeSat-Based 5G Cloud Radio Access Networks: A Novel Paradigm for On-Demand Anytime/Anywhere Connectivity," IEEE Vehicular Technology Magazine, vol. 15, no. 2, pp. 39-47, 2020. DOI: 10.1109/MVT. 2020.2979056.

[107] ETSI. (Oct. 2017). "Improved operator experience through Experiential Networked Intelligence (ENI)," [Online]. Available: https : / /www . etsi . org/images / files / ETSIWhitePapers / etsi_wp22_ENI_FINAL.pdf (visited on 2021).

[108] ETSI. (Sep. 2019). "Experiential Networked Intelligence (ENI); ENI System Architecture," [Online]. Available: https : / / www . etsi .org/deliver / etsi_gs/ENI/001_099/005/01.01.01_60/gs_ ENI005v010101p.pdf (visited on 2021).

[109] ETSI. (Dec. 2020). "Experiential Networked Intelligence (ENI); ENI requirements,” [Online]. Available: https : //www . etsi .org/deliver/etsi_ gs / ENI / 001 _ $099 / 002 / 03.01$.01_60/gs_ ENI002v030101p.pdf (visited on 2021).

[110] ETSI. (Dec. 2020). "Experiential Networked Intelligence (ENI); ENI use cases," [Online]. Available: https : / / www . etsi . org / deliver / etsi _ gs / ENI / 001 _ $099 / 001 / 03.01 .01$ _ $60 /$ gs _ ENI001v030101p.pdf (visited on 2021).

[111] ETSI. (Oct. 2016). “GANA - Generic Autonomic Networking Architecture; Reference Model for Autonomic Networking, Cognitive Networking and Self-Management of Networks and Services," [Online]. Available: https : / / www . etsi . org / images/files/etsiwhitepapers/etsi_wp16_ gana_ed1_20161011.pdf (visited on 2021).

[112] R. Bassoli, H. Boche, C. Deppe, R. Ferrara, F. H. P. Fitzek, G. Janßen, and S. Saeedinaeen, Quantum Communication Networks, 1st ed. Springer, Jan. 2021, ISBN: 978-3-030-62938-0.

[113] O. B. Akan, H. Ramezani, T. Khan, N. A. Abbasi, and M. Kuscu, "Fundamentals of molecular information and communication science," Proceedings of the IEEE, vol. 105, no. 2, pp. 306-318, Feb. 2017, ISSN: 0018-9219. DOI: 10 . 1109 / JPROC . 2016 . 2537306.

[114] J. R. Vacca, Ed., Nanoscale Networking and Communications Handbook. CRC Press, 2019, published.

[115] I. F. Akyildiz, M. Pierobon, S. Balasubramaniam, and Y. Koucheryavy, "The internet of bio-nano things," IEEE Communications Magazine, vol. 53, no. 3, pp. 32-40, Mar. 2015, ISSN: 0163-6804. DoI: 10.1109/MCOM. 2015.7060516.

\section{AUTHORS}

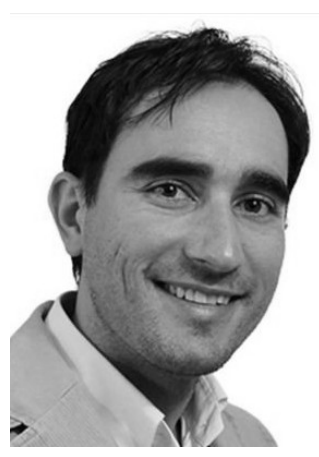

Riccardo Bassoli is a senior researcher with the Deutsche Telekom Chair of Communication Networks at the Faculty of Electrical and Computer Engineering, Technische Universität Dresden (Germany). He received his B.Sc. and M.Sc. degrees in telecommunication engineering from University of Modena and Reggio Emilia (Italy) in 2008 and 2010 respectively. Next, he received his Ph.D. degree from 5G Innovation Centre (5GIC) at University of Surrey (UK), in 2016. Between 2011 and 2015, he was also a Marie Curie Early Stage Researcher at Instituto de Telecomunicações (Portugal) and a visiting researcher at Airbus Defence and Space (France). Next, between 2016 and 2019, he was a postdoctoral researcher at the University of Trento (Italy). 


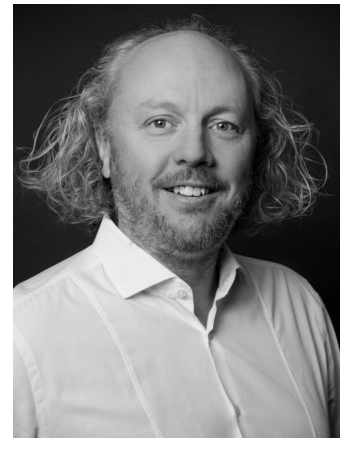

Frank H.P. Fitzek is a professor and Head of the Deutsche Telekom Chair of Communication Networks at Technische Universität Dresden (Germany), also coordinating the $5 \mathrm{G} \mathrm{Lab}$ Germany. He is the spokesman of the DFG Cluster of Excellence CeTI. He received his diploma (Dipl.-Ing.) degree in electrical engineering from the University of Technology - Rheinisch-Westfälische Technische Hochschule (RWTH), Aachen, Germany, in 1997 and his Ph.D. (Dr.-Ing.) in electrical engineering from the Technical University Berlin, Germany in 2002 and became adjunct professor at the University of Ferrara, Italy in the same year. In 2003 he joined Aalborg University as an associate professor and later became professor.

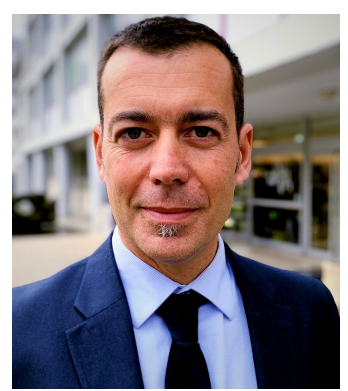

Emilio Calvanese Strinati is the 6G Program and smart devices and telecommunications scientific and innovation director at the French Atomic Energy Commission's Electronics and Information Technologies Laboratory, Grenoble, France. Since February 2021 he is also the director of the New-6G (Nano Electronic \& Wireless for $6 \mathrm{G}$ ) initiative, dedicated to the required convergence between microelectronic \& telecom, hardware \& software, network \& equipment for upcoming $6 \mathrm{G}$ technologies. His current research interests are in the area of beyond 5G future enabling technologies such as high frequency communications, mobile edge computing and distribute intelligence. 